\title{
SCARB2 Drives Hepatic Carcinoma Initiation by Supporting Cancer Stem Cell Traits and Enhancing MYC Transcriptional Activity
}

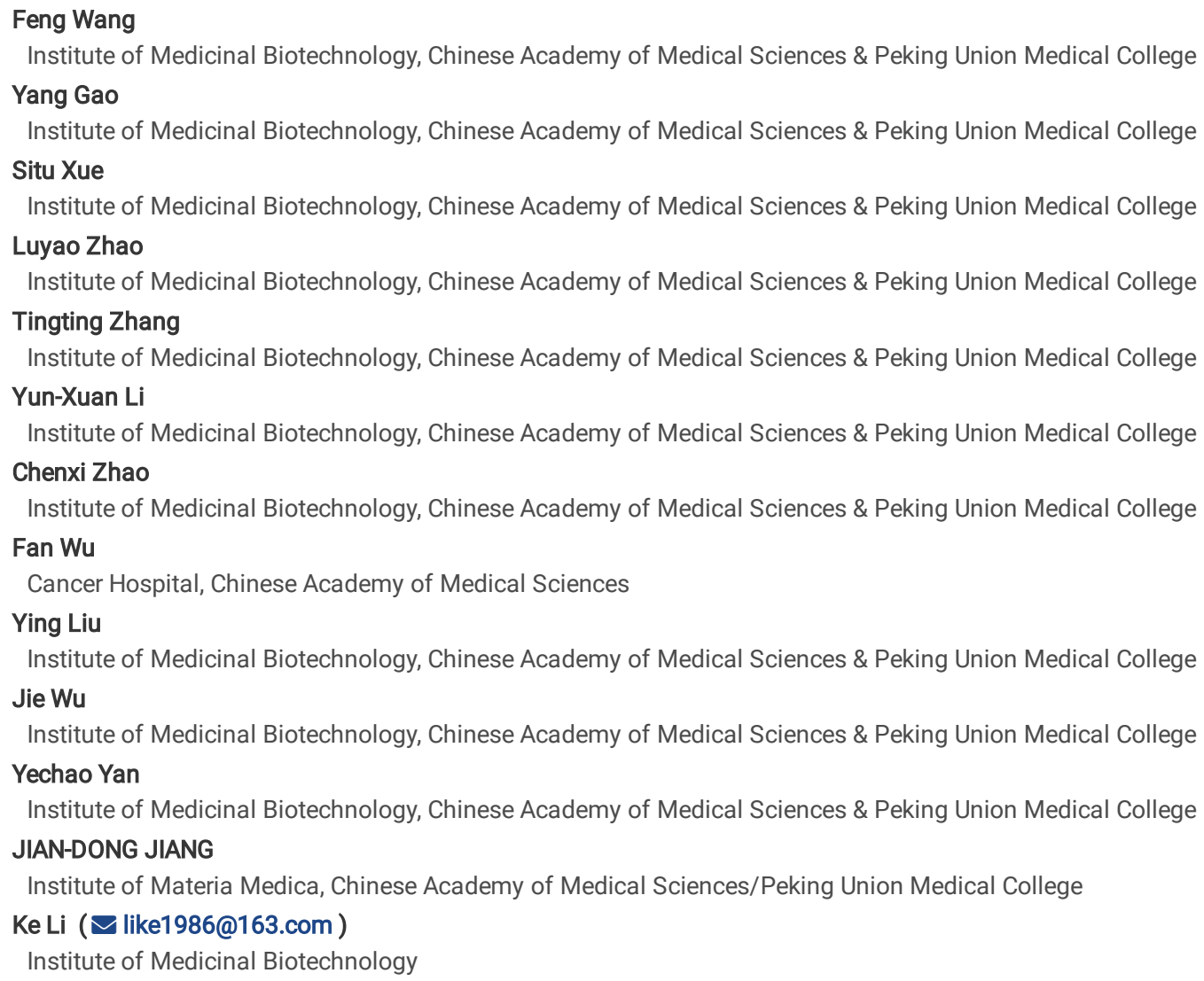

Article

Keywords: MYC, protein-protein interaction, scavenger receptor

Posted Date: February 17th, 2022

DOI: https://doi.org/10.21203/rs.3.rs-1313420/v1

License: (a) (7) This work is licensed under a Creative Commons Attribution 4.0 International License. Read Full License 


\begin{abstract}
Cancer stem cells (CSCs) with distinct metabolic features are considered to cause hepatocellular carcinoma (HCC) initiation, metastasis and therapeutic resistance. Here, we performed a metabolic gene CRISPR/Cas9 knockout screen in tumorspheres derived from HCC cells and found that deletion of the scavenger receptor SCARB2 suppressed the CSC traits of HCC cells. Using lineage tracing of the Scarb2 locus in mice, we demonstrated that Scarb2 positive $\mathrm{HCC}$ cells showed tumor-initiating activity. Specifically, Cre-mediated recombination with oncogenic MYC expression in Scarb2 positive cells drove HCC tumor formation, as determined by lineage tracing in Scarb2 mice. Knockout of Scarb2 in hepatocytes attenuated HCC initiation and progression by inhibiting CSC self-renewal. Mechanistically, binding of SCARB2 with MYC promoted MYC acetylation by interfering with HDCA3-mediated MYC deacetylation and subsequently enhanced MYC activity. Screening of a database of FDA-approved drugs showed Polymyxin B displayed high binding affinity for SCARB2 protein, disrupted the SCARB2-MYC interaction, decreased MYC activity, and reduced the tumor burden. Our study identifies SCARB2 as a marker and functional driver of HCC CSCs and suggests a targeted therapeutic option for HCC.
\end{abstract}

\title{
Introduction
}

Hepatocellular carcinoma (HCC) is the most common type of primary liver cancer and is characterized by both phenotypic and molecular heterogeneity ${ }^{1}$. The high mortality of HCC is caused by the lack of suitable biomarkers for early detection, the inadequate understanding of HCC heterogeneity, and therapeutic resistance ${ }^{2}$. Cancer stem cells (CSCs) of HCC are considered to be responsible for initiating heterogeneous tumor lesions and contributing to tumor relapse, metastasis, and therapeutic resistance ${ }^{3-5}$. Furthermore, chemotherapy and targeted therapy for HCC have been demonstrated to select for the outgrowth of therapy-resistant stem-like cancer cells, which facilitate tumor recurrence ${ }^{6,7}$. Currently, extraordinary progress has been made in the identification of liver CSCs on the basis of surface and cellular prognostic/diagnostic markers, such as cluster of CD44, CD90, CD133, CD24, and EpCAM in liver CSCs ${ }^{8-10}$. However, CSCs can also be identified in populations of cells not expressing these markers ${ }^{11}$. Thus, identifying precise markers of CSC subclones and understanding the detailed regulatory mechanism of CSC emergence and expansion provides opportunities to improve the outcomes of patients with HCC.

The proto-oncogenic transcription factor MYC is deregulated in almost all human cancers, especially HCC, and high levels of MYC are associated with poor prognosis ${ }^{12}$. Previous studies have established the contribution of MYC to the pathogenesis of liver cancer ${ }^{13,14}$ and demonstrated that activation of MYC is required for oncogenic reprogramming of adult hepatocytes into CSCs ${ }^{15}$. Thus, targeting MYC, especially in combination with traditional therapies, is considered an attractive therapeutic strategy for HCC. MYC is subject to a series of posttranslational modifications that affect its stability and oncogenic activity. To date, phosphorylation has been clearly shown to regulate MYC-mediated biological activities. Although the MYC oncoprotein is acetylated on multiple lysine residues by acetyltransferases ${ }^{16,17}$, the role of site-specific acetylation in mediating the biological functions of MYC is incompletely determined.

Scavenger receptor class B member 2 (SCARB2) belongs to the class B scavenger receptor family, which includes the receptor for selective cholesteryl ester uptake, scavenger receptor class $B$ type I (SR-BI) and CD36 ${ }^{18,19}$. A previous study indicated that both SR-BI and CD36 were more highly expressed in human HCC tissues than in the adjacent noncancerous liver tissues ${ }^{20,21}$. In particular, CSCs selectively use the scavenger receptor CD36 to promote their maintenance and mediate drug resistance in glioblastoma, leukemia and breast cancer by metabolic rewiring ${ }^{22,23}$. SCARB2 acts as a receptor in the pathogenesis of hand, foot and mouth disease ${ }^{24}$. Moreover, it functions as a phospholipid receptor ${ }^{25}$ and is involved in lysosomal cholesterol export ${ }^{19}$ However, the role of SCARB2 in cancer, especially HCC, remains uncharacterized. In this study, we investigated and verified the role of SCARB2 in hepatic CSC biology and HCC initiation and progression by using CRISPR/Cas 9 screening and in vivo lineage tracing approaches. Our results established a direct link between SCARB2+ HCC cells and CSC properties. We further demonstrated that SCARB2 interacts with MYC to interfere with HDCA3-mediated MYC deacetylation. In addition, we evaluated the therapeutic potential of targeting SCARB2 in HCC. Taken together, this study reveals SCARB2 as a new regulator of CSCs and a potential new therapeutic target in HCC.

\section{Results}

\section{CRISPR/CAS9 library screening identified SCARB2 as a critical gene for maintaining the stem cell-like characteristics of HCC cells}

CSCs have a different metabolic phenotype to that of differentiated bulk tumor cells, and these specific metabolic activities directly participate in the process of tumor transformation. To identify critical metabolic genes involved in supporting CSC traits in human HCC, we performed a human CRISPR/Cas9 metabolic gene knockout library screen in HCC cells precultured in ultra-low attachment dishes. HCC tumorspheres were transduced with the lentiviral constructs in the human CRISPR/Cas 9 metabolic gene knockout library. After 15 days of culture, the numbers of wells containing a tumorsphere larger than $70 \mu \mathrm{m}$ or smaller than $50 \mu \mathrm{m}$ were determined using a phase contrast microscope (Fig. 1a-b; Extended Data Fig. 1a). For several gene targets, multiple sgRNAs targeting the same gene were enriched in selected small tumorspheres, and the 10 most significant hits were shown as Fig. 1c.

To verify the results of the stemness screen, we validated each of the top 10 genes. Most screened sgRNAs that targeted these 10 genes decreased the tumorsphere formation capacity of HCC cells (Extended Data Fig. 1b). In particular, SCARB2 deletion (Extended Data Fig. 1c) suppressed tumorsphere formation in $\mathrm{HCCML} 3$, HepG2, and primary human $\mathrm{HCC}$ cells $\left(\mathrm{HCC}^{1 \#}\right)$ freshly isolated from patient-derived tumors (Fig. 1d) and decreased the proliferation of HCCLM3 and HepG2 cells (Fig. 1e). HCC stem cells are suggested to cause drug resistance and metastasis due to their ability to self-renew and differentiate into heterogeneous lineages of cancer cells ${ }^{4}$. Tumor organoids with SCARB2 deletion (Extended Data Fig. 1c) showed a higher apoptosis ratio than their control counterparts (Fig. 1f). Furthermore, loss of SCARB2 not only decreased the invasion of HepG2 cells but also sensitized HCCLM3 and HepG2 cells to 
sorafenib (Fig. 1g-h). Interestingly, the analysis of the HCC patient data from The Cancer Genome Atlas (TCGA) revealed that there was positive correlation between SCARB2 expression with known CSC markers (CD133, EPCAM or CD24) (Fig. 1i). In addition, SCARB2 expression was enhanced in tumor compared to that in normal liver tissues and up-regulation of SCARB2 was observed in every stage of HCC patients (Fig. 1j-k). We further analyzed the protein level of SCARB2 in HCC specimens using tissue microarray and immunohistochemistry. Compared with normal liver tissues, most HCC specimens showed high expression of SCARB2 (Fig. 1l-m). Moreover, high expression of SCARB2 was associated with short survival times in patients with HCC (Fig. 1n). These data suggest that SCARB2 may perform critical pathogenic functions by supporting stem cell-like characteristics in HCC cells.

\section{SCARB2+ HCC cells are tumor-initiating cells (TICs) and drive the expansion of tumor cells with stem cell characteristics}

To explore the role of SCARB2 in vivo, we first engineered a mouse strain expressing inducible CreERT2 recombinase and EGFP from the endogenous Scarb2 locus and CreERT2 knockin did not affect the endogenous SCARB2 expression (Extended Data Fig. 2a). Diethylnitrosamine (DEN) was injected into Scarb2 ${ }^{\text {CreERT2-EGFP }}$ knock-in mice ${ }^{26}$, and this model was further used to examine the function of Scarb2+ (Scarb2 ${ }^{\text {EGFP+ }}$ ) cells in HCC (Fig. 2a). A subset of cells throughout the normal liver expressed EGFP and the hepatocyte marker ALB in Scarb2 CreERT2-EGFP mice (Fig. 2b left). Less EGFP expression was detected in blood cells and other liver cell types, including kupffer cells, stellate cells and endothelial cells (Extended Data Fig. 2b). Furthermore, the ratio of Scarb2 ${ }^{\text {EGFP+ }}$

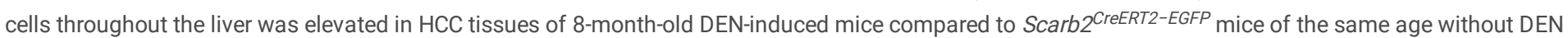
injection (Fig. 2b). We next isolated Scarb2 ${ }^{\mathrm{EGFP}+} \mathrm{HCC}$ cells from liver cancer tissue of 8-month-old Scarb2 ${ }^{\text {CreERT2-EGFP }}$ mice by fluorescence-activated cell sorting (FACS). Scarb2 ${ }^{\text {EGFP+ }}$ HCC cells formed substantially more and larger colonies than Scarb2-negative (Scarb2 ${ }^{\text {EGFP- }}$ ) HCC cells (Fig. 2c). Moreover, we found that the Scarb2 ${ }^{\text {EGFP+ }}$ population was comprised of $\mathrm{CD} 24+$ stem cell populations and a CD133+ progenitor population with a strong HCC tumor-initiating ability (Extended Data Fig. 2c). In particular, Scarb2 ${ }^{\mathrm{EGFP}+} \mathrm{HCC}$ cells exhibited a higher capacity to form secondary tumors in recipient mice in a limiting dilution assay (LDA) to assess the tumor repopulation ability (Fig. 2d). We further sorted SCARB2+ and SCARB2- cells from HCC clinical samples and test if they also display CSC properties. As shown in Fig. 2e, SCARB2+ HCC cells formed more and larger colonies than SCARB2- HCC cells. These data indicate that Scarb2+ HCC cells have characteristics of the TIC population.

To further verify whether Scarb2+ cells are HCC-initiating cells, we used lineage tracing analysis to determine the cell origin of transformed cells in mouse HCC models. Scarb2 ${ }^{\text {CreERT2-EGFP }}$ mice were crossed with Rosa26 $6^{\text {LLL-Tomato/+ }}$ reporter mice; this modification enables permanent cell labeling by deletion of a transcriptional stop element flanked by loxP sites and concomitant expression of the fluorescent tomato protein (Extended Data Fig. 2d). The resulting Scarb2 ${ }^{\text {CreERT2-EGFP }}$ 26 Tomato mice were then crossed with H11-CAG-LSL-Myc mice (Extended Data Fig. 2e; Fig. 2f) to induce overexpression of the oncogene MYC in Scarb2+ cells. These Scarb2 ${ }^{\text {CreERT2-EGFP }}$ 26 $6^{\text {Tomato }}$ Myc mice (2 weeks old) were injected with tamoxifen (TAM) and analyzed in different times (Fig. 2f). Tomato-positive (tomato ${ }^{+}$) cells composed $8.9 \pm 0.5 \%$ of the liver area in Scarb2 ${ }^{\text {CreERT2-EGFP }}{ }^{2} 26^{\text {Tomato }}$ Myc mice 7 days after tamoxifen treatment, and the area composed of tomato+ progeny increased progressively during the tracing period to $11.2 \pm 2.2 \%$ of the liver area at 1 month, $34.3 \pm 9.7 \%$ of the liver area

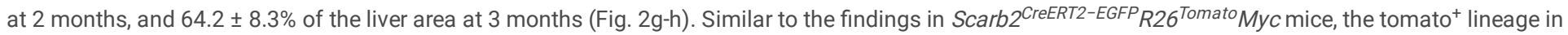
tamoxifen-injected Scarb2 ${ }^{\text {CreERT2-EGFP }}$ R2 $6^{\text {Tomato }}$ mice accounted for $6.8 \pm 0.3 \%$ of the cells at 7 days and increased over time (8.2 $\pm 0.5 \%$ at 1 month, $20.2 \pm$ $2.5 \%$ at 3 months and $32.7 \pm 4.9 \%$ at 6 months) (Extended Data Fig. 2f). Most tomato ${ }^{+}$cells in Scarb2 ${ }^{\text {CreERT2-EGFP }}{ }^{R 26^{\text {Tomato }}}{ }^{\text {Myc mice and }}$ Scarb2 ${ }^{\text {CreERT2-EGFP }}{ }^{2} 26^{\text {Tomato }}$ mice detected after 3 months of tamoxifen treatment were HNF4A+ hepatocytes (Fig. 2i-j; Extended Data Fig. 2g), and tomato ${ }^{+}$ cells were not detected in these two strains of mice treated with oil vehicle (Extended Data Fig. 2h). In addition, we observed that the Scarb2-positive cells differentiated into most hepatocytes and some biliary epithelium cells in liver tissues from Scarb2 ${ }^{\text {CreERT2-EGFP }}{ }^{R 26^{\text {Tomato }}}$ mice, which was demonstrated by the colocalization of tomato ${ }^{+}$cells (Scarb2-positive's daughter cells) with HNF4A ${ }^{+}$hepatocytes or CK19+ biliary epithelium cells (Fig. $2 \mathrm{k}$ left). However, the differentiation ability of the Scarb2-positive cells into biliary epithelium cells increased when MYC expression was driven in Scarb2-positive cells from Scarb2 ${ }^{\text {CreERT2-EGFP }}$ R26 ${ }^{\text {Tomato }}$ MYC mice (Fig. $2 \mathrm{k}$ right). These data indicate that rare Scarb2 ${ }^{+}$cells drive a marked and progressive repopulation of the hepatocyte lineage and show differentiation ability into hepatocyte and biliary epithelium cell types during homeostasis.

Interestingly, 30 days after treatment, histological analysis revealed precancerous hepatic foci in the livers of tamoxifen-treated Scarb2 ${ }^{\text {CreERT2-EGFP }}$ R26 ${ }^{\text {Tomato }}$ Myc mice compared to vehicle-treated mice (Fig. 2l left). In addition, approximately $60 \%$ of tamoxifen-treated Scarb2 ${ }^{\text {CreERT2-EGFP }}$ R2 $6^{\text {Tomato }}$ Myc mice exhibited abdominal swelling after 60 days of tamoxifen treatment (Fig. $2 \mathrm{~m}$ ). Many tumor nodules were found on the surface of the liver in tamoxifen-treated mice (Fig. 2n), and hematoxylin and eosin (HE) staining revealed numerous tumor foci in liver tissue sections from tamoxifen-treated Scarb2 ${ }^{\text {CreERT2-EGFP }}{ }^{2} 26^{\text {Tomato }} \mathrm{MyC}$ mice but not in those from vehicle-treated mice (Fig. 21 right). During the 2-month observation period after tamoxifen treatment, $75 \%$ of Scarb2 ${ }^{\text {CreERT2-EGFP }}{ }^{2} 26^{\text {Tomato }}$ Myc mice exhibited HCC characteristics (Fig. 20). These data suggested that SCARB2 ${ }^{+} \mathrm{HCC}_{\text {cells }}$ function as liver TICs to drive HCC carcinogenesis.

\section{SCARB2 deletion suppresses the initiation and progression of HCC by reducing MYC activity}

To further investigate the role of SCARB2 in controlling the TIC activity of HCC cells, we generated mice with liver-specific conditional Scarb2 knockout (Extended Data Fig. 3a) by crossing Scarb2-floxed $\left(S c a r b 2^{F / F}\right)$ mice and mice with hepatocyte-specific expression of Cre recombinase $\left(C r e^{A l b}\right)($ Extended Data Fig. 3b). Abrogation of SCARB2 expression was confirmed by the reduction in the SCARB2 protein level in the hepatocytes of mice with homozygous deletion of SCARB2 (Extended Data Fig. 3c). The body and liver weights of 10-week-old Cre ${ }^{A / b} S c a r b 2^{F / F}$ mice were comparable to those of the control WT mice (Extended Data Fig. 3d). Macroscopically, Scarb2 KO livers appeared normal (Extended Data Fig. 3e). There was no significant difference in serum alanine

Page $3 / 21$ 
aminotransferase (ALT), aspartate aminotransferase (AST), cholesterol (CHO), glucose (GLU), and triglyceride (TG) levels for these two groups (Extended Data Fig. 3e-f), indicating that deletion of Scarb2 didn't affect liver homeostasis. These Cre ${ }^{A l b} S c a r b 2^{F / F}$ mice were further crossed with H11-CAG-LSL-MyC mice to generate heterozygous Scarb2 knockout $C r e^{A / b} S c a r b 2^{F /+} M y c$ mice and homozygous Scarb2 knockout Cre ${ }^{A / b} S c a r b 2^{F / F} M y c$ mice (Fig. 3a, Extended Data Fig. 3g). After mating H17-CAG-LSL-Myc mice with Cre ${ }^{A l b}$ mice, liver cancer developed spontaneously in 6- to 8-week-old Cre ${ }^{A / b} \mathrm{Myc}$ mice (Fig. 3b-c). Heterozygous knockout of Scarb2 decreased the tumor size (Fig. 3c). The tumor size was further decreased in $\mathrm{Cre}^{A l b} S c a r b 2^{\mathrm{F} / F} \mathrm{Myc}$ mice (Fig. 3b-c). Furthermore, even with heterozygous knockout of Scarb2, the tumor incidence rate decreased, and the survival time of $C r e^{A l b} S c a r b 2^{F /+} M y c$ mice was extended based on KaplanMeier analysis (Fig. 3d-e). Cre ${ }^{A / b} S c a r b 2^{F / F} M y c$ mice showed a significantly lower incidence of HCC and longer survival times than $C r e^{A / b} S c a r b 2^{F /+} M y c$ mice and $\mathrm{Cre}^{A / b} \mathrm{Myc}$ mice (Fig. 3d-e). Consistent with these results, a LDA to assess the tumor repopulation ability revealed that tumor cells from $C r e^{A l b} S c a r b 2^{F / F} M y c$ and $C r e^{A l b} S c a r b 2^{F /+} M y c$ mice exhibited reduced stem cell activity (Fig. 3f). To confirm the inhibitory effect of SCARB2 deletion on HCC progression, we knocked out SCARB2 in human HepG2 HCC cells using lentivirus-mediated delivery of CRISPR-Cas9 system components. SCARB2 deletion impeded the growth (Fig. $3 \mathrm{~g}$ ) and decreased the weight (Fig. 3h-i) of tumors in mice inoculated with HepG2 cells. Indeed, SCARB2 deletion also decreased the number of lung metastatic nodules formed by HCCLM3 cells (Fig. 3j-k). Collectively, these data suggest that SCARB2 positively regulates the CSC-like traits and invasiveness of HCC cells.

To investigate the mechanism by which SCARB2 promotes HCC pathogenesis, we analyzed the gene expression profiles of HCC cells with or without Scarb2 deletion through RNA sequencing (RNA-seq) (Fig. 4a). Gene set enrichment analysis (GSEA) showed high enrichment of MYC target genes in HCC cells from $\mathrm{Cre}^{A / b} \mathrm{Myc}$ mice compared to HCC cells from $\mathrm{Cr}^{A / b} S c a r b 2^{F / F} \mathrm{Myc}$ mice (Fig. 4b). Single-cell RNA sequencing of human primary HCC tumorspheres indicated that SCARB2 positive cells accounted about $11.3 \%$ of total tumorspheres and were enriched with MYC target genes (Fig. 4c-e). Most target genes are activated by MYC in an E-box-dependent manner. By using a luciferase reporter construct containing four E-boxes, we observed that SCARB2 depletion decreased MYC activity (Fig. 4f) in HCC cells, while SCARB2 overexpression had the opposite effect (Fig. 4g). Consistent with these findings, qRT-PCR analysis showed that Scarb2 deletion suppressed the transcription and translation of several critical MYC target genes in both mouse- and human-derived HCC cells (Fig. 4h-i; Extended Data Fig. 4). We next performed chromatin immunoprecipitation with high-throughput sequencing (ChIP-seq) in HCC cells with or without SCARB2 deletion. ChIP-seq showed that SCARB2 deletion globally reduced the association of MYC with the promoters of its target genes (Fig. 4j-I). The analysis of a publicly available HCC patient data from TCGA revealed that there was positive correlation between SCARB2 with MYC target genes (GLS, CDK4, LDHA, SLC2A, CCNA1, PLD6, CD133, EPCAM or EIF3A (Fig. 4m). Taken together, these data indicate that SCARB2 enhances HCC CSC self-renewal by enhancing MYC transcriptional activity and increasing the expression of its target genes.

\section{SCARB2 interacts with MYC to disrupt HDAC3-mediated MYC deacetylation}

Posttranslational modifications, including phosphorylation, ubiquitylation, and acetylation, play critical roles in controlling MYC expression and activity. SCARB2 overexpression did not affect the total phosphorylation level of MYC on serine (Ser, S) and threonine (Thr, T) residues (Extended Data Fig. 5a-b) or the ubiquitylation of MYC (Extended Data Fig. 5c). However, SCARB2 deletion obviously reduced the level of MYC acetylation (Fig. 5a), and SCARB2 overexpression had the opposite effect (Fig. 5b). We further explored the detailed mechanism by which MYC acetylation contributes to the regulation of MYC transcriptional activity by SCARB2. Quantitative mass spectrometry (MS)-based proteomics was used to assess acetylation modification of MYC in HCCLM3 cells with or without SCARB2 overexpression. Peptides with acetylation on lysine (K) 148 and 326 of MYC were enriched in SCARB2-overexpressing HCCLM3 cells (Supplementary table 1). To further confirm the trends observed in our mass spectrometry-based analysis, we next mutated K148 and K326 to the nonacetylatable amino acid arginine (R) and quantified the acetylation level of the MYC K148R and K326R mutants in HEK293 cells with or without SCARB2 overexpression. Compared to wild-type MYC, both the K148R and K326R MYC mutants exhibited reduced acetylation (Fig. 5c; Extended Data Fig. 5d). However, the K148R mutation but not the K326R mutation diminished the effect of SCARB2 overexpression on MYC acetylation (Fig. 5c; Extended Data Fig. 5d). These data indicate that SCARB2 upregulates MYC acetylation on K148.

p300 ${ }^{16}$, HDACs ${ }^{27}$, KAT5 $^{28}$, SIRT $1{ }^{29}$, and GCN5 ${ }^{30}$ affected MYC acetylation and deacetylation in both direct and indirect manners. We found that SCARB2 overexpression further increased MYC acetylation in p300-, KAT5-, or GCN5 silencing HCC cells (Extended Data Fig. 5e-g), suggesting that one or more additional acetyltransferases or deacetylases may be involved in this SCARB2-regulated process. However, the decrease in MYC acetylation mediated by the deacetylase HDAC3 but not SIRT1 was restored by overexpression of SCARB2 in HCC cells (Fig. 5d; Extended Data Fig. 5h), indicating that SCARB2 affects MYC acetylation mainly in an HDAC3-dependent manner. We next applied CRISPR/Cas9 gene editing to generate $M Y C^{148 R}$ mutant HCCLM3 cell lines with or without SCARB2 deletion (Fig. 5e). The K148R mutation not only reduced MYC acetylation but also diminished the effect of HDAC3 overexpression on MYC deacetylation (Fig. 5f). Moreover, the K148R mutation decreased the transcriptional activity of MYC compared to that of MYCWT; However, SCARB2 deletion only reduce MYC transcriptional activity in $M Y C^{W T}$ but not $M Y C^{K 148 R}$ HCCLM3 cells (Fig. $5 \mathrm{~g}$ ). Given that K148, located in the CT domain of MYC, mediates the interaction of MYC with several cofactors, including GCN5, KAT5, and BRD $4{ }^{31}$, we examined whether acetylated K148 of MYC is the binding site between MYC and these cofactors. Endogenous coimmunoprecipitation (Co-IP) indicated that the K148R mutation impeded the ability of MYC to interact with BRD4, KAT5, and GCN5 (Fig. 5h). Taken together, these data suggest that K148 acetylation of MYC is critical for MYC transcriptional activity by providing the docking site for critical cofactors with MYC (Fig. 5i).

We next explored the molecular mechanism of the SCARB2 protein in HDAC3-mediated MYC deacetylation. Deletion of SCARB2 increased but overexpression of SCARB2 reduced the interaction of HDAC3 with MYC (Fig. 5j; Extended Data Fig. 5i). SCARB2 interacts with the CT element and C-terminal domain (CTD) of MYC (Fig. 5k), the same regions responsible for the interaction of MYC with HDAC3 (Fig. 5I). Moreover, using SCARB2 mutants, we identified domains involved in the interaction between SCARB2 and MYC. Deletion of the C-terminal transmembrane domain (CTM), N-terminal transmembrane domain (NTM) or cytoplasmic domain (CD) did not affect the interaction of SCARB2 and MYC, but luminal domain (LD) deletion significantly decreased the interaction of 
SCARB2 and MYC, indicating that the LD domain is the primary contributor to this interaction (Fig. 5m). Taken together, these results suggest that SCARB2 competes with HDAC3 to bind with MYC, which in turn elevates MYC acetylation.

\section{Polymyxin B (PMB) binds with SCARB2 to counteract HCC stemness and progression}

Repurposing of already-approved drugs for other indications may be an alternative strategy for drug development. We next performed virtual screening and binding assays using AutoDock Vina, a docking program that computationally examines the binding energy of a compound to its target, to identify small molecules that target the LD domain of SCARB2 (Fig. 6a). We ranked 1317 Food and Drug Administration (FDA)-approved drugs according to their simulated binding energy. We then selected the top 10 compounds with a docking energy $\leq-10.0 \mathrm{kcal} / \mathrm{mol}$ (Fig. $6 \mathrm{~b}$ ) and examined the binding abilities of these 10 candidate SCARB2 inhibitors using surface plasmon resonance (SPR) analysis. Four drugs-Fertilan, gallotannic acid, PMB, and alarelin-exhibited binding to SCARB2 (Extended Data Fig. 6; Fig. 6C). Among these drugs, PMB displayed a high binding affinity for SCARB2 ( $K_{D}=2.82 \mu M$; Fig. $\left.6 \mathrm{C}\right)$. Specifically, prediction of the interacting residues of SCARB2 with PMB showed several hydrogen bonds between the LD domain of the SCARB2 protein and PMB (Fig. $6 \mathrm{~d}$ ). Furthermore, PMB decreased the binding affinity between SCARB2 and MYC in vitro (Fig. 6e). High-resolution structured illumination microscopy (SIM) showed the SCARB2-MYC colocalization in HCC cells treated with vehicle (CON), whereas PMB inhibited the colocalization and interaction of SCARB2 with MYC (Fig. 6f-g) and rescued the binding of MYC with HDAC3 (Fig. 6h). Furthermore, PMB reduced MYC acetylation and transcriptional activity in HCC cells (Fig. 6i-j). RNA-seq showed that MYC target gene sets were enriched in CON-treated HCC cells (Fig. 6k). PMB treatment decreased the tumorsphere formation capacity and led HCCLM3 cells hypersensitize to sorafenib (Fig. 6l). In human HCC tumor organoids, sorafenib treatment induced apoptosis, and this effect was further enhanced when PMB was combined with sorafenib (Fig. 6m). In addition, the combination of PMB with sorafenib synergistically inhibited the proliferation of several HCC cell lines (Fig. 6n). We further used MYC or SCARB2 depleted HCC cells to examine the effects of PMB on cell growth and colony formation ability. MYC or SCARB2 depletion diminished the anti-proliferation and anti-stemness ability of PMB treatment (Fig. 6o-r), suggesting the specificity of PMB to SCARB2/MYC cascade. These data indicate that PMB disrupts the SCARB2-MYC interaction and subsequently decreases MYC acetylation and activity to counteract $\mathrm{HCC}$ progression.

We further examined the antitumor effect of PMB in cell line-derived xenograft (CDX) models. PMB treatment showed no obvious side effects in the HCC mouse models, as demonstrated by the no significant difference in body/liver weights, serum ALT, AST, CHO, GLU, or TG levels of HCC mice with or without PMB treatment (Extended Data Fig. 7a-c). PMB suppressed the growth of HepG2 and HCCLM3 tumors (Fig. 7a-b), and the combination of PMB and sorafenib showed a marked inhibitory effect on HepG2 and HCCLM3 tumor growth (Fig. 7a-c). In addition, PMB reduced the TIC frequency from 1 in $\sim 121$ cells in tumors from CON-treated HepG2 tumor-bearing mice to 1 in $~ 1474$ cells in tumors from PMB-treated mice (Fig. 7d top). Similarly, PMB treatment reduced the capacity of HCCLM3 cells to form secondary tumors (Fig. 7d, bottom). Moreover, sorafenib reduced the TIC frequency in mice bearing HepG2 and HCCLM3 CDXs by 6-fold, and these effects were more significant in mice treated with the combination of PMB and sorafenib (Fig. 7d). Mechanistically, PMB reduced the acetylation of MYC through disrupting the interaction between SCARB2 and MYC in HepG2 and HCCLM3 tumors (Fig. 7e-f). In an established HCC patientderived xenograft (PDX) model with positive expression of MYC and SCARB2 (Fig. 7g-h), we observed that PMB suppressed the tumor growth and the combination of PMB and sorafenib further decreased the growth and weights of PDXs in mice (Fig. 7i-k). PMB decreased ALDH ${ }^{+}$subpopulation ratio of PDX tumors with or without sorafenib treatment (Fig. 7l). These data indicate that targeting SCARB2 may serve as a therapeutic strategy for HCC by reducing the number and inhibiting the function of HCC-initiating cells.

\section{Discussion}

Although targeting CSCs has been considered a promising strategy for the treatment of HCC by suppressing tumor initiation, metastasis, and chemotherapeutic resistance 3,5 , relatively little is known regarding the therapeutic targets that control TIC activity and contribute to HCC occurrence and progression. In this study, we observed that SCARB2 expression was enhanced in human HCC samples compared with normal liver tissues. Using lineage tracing of Scarb2 in mice, we identified that Scarb2+ HCCs have the stem cell trait of tumor initiation capability. Scarb2+ cells drove the formation of HCC tumors and Scarb2 knockout suppressed the proliferation and self-renewal ability of these HCC cells by reducing MYC transcriptional activity and acetylation. Our study thus provides insights into the function and mechanism of CSCs and targeted anti-CSC therapies in HCC.

The existence of liver CSCs has been explored by identification and classification using normal liver stem/progenitor cell markers, such as EpCAM, Lgr5, CD133, and CD24 26,32,33, but the origins of liver CSCs are not well established ${ }^{3,34}$. Tumor transplantation and lineage tracing analysis are the current "gold standard" approaches for characterizing and identifying the cell of origin of CSCs because they can assess the self-renewal, multipotency, proliferative potential and fate of stem cells during tumor formation ${ }^{35}$. In this study, we established a bigenic mouse line, Scarb2 ${ }^{\text {CreERT2-GFP }}$ R26 ${ }^{\text {Tomato }}$, to achieve specific labeling of Scarb2+ cells (GFP+) and their daughter cells (tomato+). Scarb2+ liver cells possess the property of potential liver stem/progenitor cells to produce additional hepatocytes during liver development. Using DEN treatment of Scarb2 ${ }^{\text {CreERT2-GFP }}$ mice and MYC-driven reprogramming to introduce oncogenic events specifically into Scarb2+-cells in vivo, we identified Scarb2+ HCC cells as CSCs with high tumor-initiating ability. Notably, comparable adenomas were generated by using the Cre-ERT2 driver to induce MYC gene expression in these Scarb2+ cells. Although Scarb2+ cells (GFP+) accounted for only approximately $10 \%$ of the cells in HCC tumors, most cells in the entire tumor were daughter cell label+ (i.e., tomato+), suggesting that the specific Scarb $2+$ cell population can function as an origin of cancer cells. Collectively, our study provided direct and functional evidence for visualizing and monitoring the presence and fate of Scarb2+ CSCs during HCC development in mouse model.

Recently, the role of MYC acetylation and deacetylation has attracted much attention due to its effect on the regulation of MYC expression and/or function through transcriptional and posttranscriptional mechanisms ${ }^{17,36}$. Previous studies have established a link between MYC and HDAC3 ${ }^{27,37}$. K143 and K157 of MYC are the sites of HDAC3-mediated deacetylation, and TRAF6 regulates MYC stability through HDAC3-mediated K143 and K157 deacetylation ${ }^{38}$. However,

Page 5/21 
in this study, we found that SCARB2 supports HCC stem cell-like features by interacting with MYC, which subsequently impairs HDAC3-mediated MYC deacetylation at K148 and thereby enhancing MYC transcriptional activity. Our results revealed that K148 is an additional HDAC3-mediated deacetylation site in MYC. K148 acetylation and deacetylation affect MYC transcriptional activity mainly by interfering with the recruitment of cofactors to MYC, but not influencing MYC stability.

The MYC protein interactome impacts many of the biological functions related to MYC, offering several opportunities for therapeutic intervention ${ }^{39,40}$. Our previous study showed that an alpha-helical peptide interfering with the protein-protein interaction (PPI) of MYC and TRIB3 displayed anti-lymphoma efficacy both in vitro and in vivo ${ }^{41}$. These results are encouraging and validate the quest to target MYC PPIs to diminish its oncogenic function. In this study, we verified that the MYC-SCARB2 PPI promotes the oncogenic function of MYC by interfering with HDAC3-mediated deacetylation of MYC. More importantly, we performed a screen and identified a SCARB2 binding molecule, PMB, which is a clinically available polymyxin antibiotic that has re-emerged clinically to treat infections caused by multidrug- or extensively drug-resistant gram-negative bacteria. To date, few studies have reported that PMB has antitumor effects in the clinic or on the bench. We identified PMB as a SCARB2 binding molecule that interferes with the PPI of SCARB2 and MYC, thereby reducing MYC acetylation and transcriptional activity. PMB treatment alone or in combination with sorafenib suppressed HCC progression in several mouse models. In summary, our study indicates that SCARB2, particularly in the context of the SCARB2-MYC PPI, is a potential therapeutic target for HCC. Furthermore, our results encourage immediate clinical translation of PMB-based therapeutics for HCC treatment.

\section{Methods Animal studies}

NOD-scid IL2Rg null (NSG) mice (4-6 weeks old, male) were purchased from Shanghai Nan Fang Model Biotechnology Co. Ltd. (Shanghai, China). BALB/c nude mice and C57BL/6 mice were purchased from Beijing Hua Fu Kang Bioscience Co., Ltd. (Beijing, China). Cre ${ }^{A l b}$ (B6.Cg-Speer6-ps1Tg(Alb-cre)21Mgn/J) mice were obtained from from Cyagen Biosciences Inc. (Guangzhou, China). Scarb2 knockout (Scarb2loxP/loxP, Scarb2 ${ }^{\mathrm{F} / \mathrm{F}}$ ) mice were generated by Cyagen Biosciences Inc. (Guangzhou, China). The gRNA for the mouse Scarb2 gene, the donor vector containing loxP sites, and Cas 9 mRNA were coinjected into fertilized mouse eggs to generate offspring with targeted conditional knockout. Mice with hepatocyte-specific conditional Scarb2 knockout were generated by crossing Scarb2 ${ }^{F / F}$ mice with Cre ${ }^{A / b}$ mice. Scarb2 ${ }^{C r e E R T 2-E G F P}$ transgenic mice were generated by GemPharmatech Biotechnology Co., Ltd. (Nanjing, China). Scarb2 ${ }^{\text {CreERT2-EGFP }}$ mice were bred with mice of the ROSA26-CAG-LSL-tdTOMATO (R26 $6^{\text {Tomato }}$ ) reporter line (GemPharmatech Co., Ltd., Nanjing, China) to generate Scarb2 ${ }^{\text {CreERT2-EGFP }}$ 26 $6^{\text {Tomato }}$ mice for lineage tracing analysis. Myc/F (C57BL/6-Igs2em1(CAG-LSL-Myc)Smoc) mice were obtained from the Shanghai Research Center for Model Organisms (Shanghai, China). The conditional overexpression sequence with the CAG promoter-loxp-STOP-loxp-MycpolyA structure was inserted into the H11 locus to establish the H11-LSL-Myc mouse model.

All mice were maintained in the animal facility at the Institute of Materia Medica under specific pathogen-free (SPF) conditions. Mice were housed in groups of 4-6 in individually ventilated cages on a $12 \mathrm{hr}$ light/dark cycle (07:30-19:30 light, 19:30-07:30 dark) in a room with controlled temperature $\left(23 \pm 2{ }^{\circ} \mathrm{C}\right)$ and relative humidity (40-50\%). For animal studies, mice were earmarked before grouping and were then randomly separated into groups by an independent person; however, no particular method of randomization was used. All animal studies were approved by the Animal Experimentation Ethics Committee of the Chinese Academy of Medical Sciences (Permit No. IMB-20190423D702), and all procedures were conducted in accordance with the guidelines of the Institutional Animal Care and Use Committees of the Chinese Academy of Medical Sciences. The animal study was conducted in accordance with the Animal Research: Reporting of In Vivo Experiments (ARRIVE) guidelines.

\section{CRISPR/Cas9 knockout library screen}

Human CRISPR Metabolic Gene Knockout library was a gift from David Sabatini (Addgene \#110066). The workflow of this forward genetic screen is illustrated in Fig. 1a. First, HEK293FT cells were seeded at $15 \mathrm{~cm}$ culture dish (Corning Inc., \#430599) for library scale production. Cells were transfected the next day at 80-90\% confluency. $2.7 \mu \mathrm{g}$ of pMD2.G (Addgene), $5.4 \mu \mathrm{g}$ of psPAX2 (Addgene), $10.7 \mu \mathrm{g}$ of lentivirus target plasmid (Addgene \#110066) were transfected using $500 \mu \mathrm{l}$ of DMEM and $877 \mu \mathrm{l}$ of prepare polyethylenimine (PEI, Polysciences Inc., \#23966). $5 \mathrm{~h}$ after transfection the media was changed. Virus supernatant was harvested $48 \mathrm{~h}$ post-transfection, filtered with a 0.45-mm PVDF filter (Millipore), aliquoted, and stored at -80 C.

HCCLM3 cell were precultured in ultra-low attachment plate for 7 days. Then we transduced these HCCLM3 cells with lentivirus of Metabolic Gene Knockout library which contains targets 2,981 human metabolic genes with 29,790 guide RNAs at a low MOI ( 0.3) to ensure effective barcoding of individual cells. Then, the transduced cells were selected with $1.6 \mu \mathrm{g} / \mathrm{ml}$ of puromycin for 7 days to generate a mutant cell pool. After selection for transduced cells, 5,000 cells of the mutant cell pool were precisely transferred to an ultra-low attachment 96 -well plate at a ratio of 1 single viable cell per well. After 15 days of culture, Genomic DNA was extracted from the selected small tumorsheres (diameter $<50 \mu \mathrm{m}$ ) and big tumorsheres (diameter $>70 \mu \mathrm{m}$ ), and the sgRNA sequences were amplified using NEBNext ${ }^{\circledR}$ High-Fidelity 2X PCR Master Mix and subjected to massive parallel amplicon sequencing carried out by Novogene Technology (Beijing, China). The sgRNA read count and hits calling were analyzed by MAGeCK v0.5.7 algorithm.

\section{Isolation and culture of tumor organoids}

The noncancerous segment of the liver cancer tissue was removed, and liver cancer cells were isolated through a combination of mechanical disruption and enzymatic digestion. In brief, patient-derived or mouse $\mathrm{HCC}$ tissue of the appropriate size was minced, washed and incubated at $37^{\circ} \mathrm{C}$ with digestion solution containing $1-2 \mathrm{mg} / \mathrm{ml}$ collagenase (Sigma, C9407) on an orbital shaker at $37^{\circ} \mathrm{C}$ for $2-5 \mathrm{hr}$. The suspension was strained through a $100 \mu \mathrm{m}$ nylon cell strainer to retain tissue fragments. The cell pellet was obtained after centrifugation at $400 \mathrm{~g}$ for $5 \mathrm{~min}$. The pellet was washed in cold advanced DMEM/F12 and was then mixed with $10 \mathrm{mg} / \mathrm{ml}$ cold Cultrex growth factor-reduced basement membrane extract (BME, Type 2, Pathclear) and allowed to solidify on prewarmed 24well suspension culture plates at $37^{\circ} \mathrm{C}$ for $30 \mathrm{~min}$. After the BME was solidified, the samples were cultured in classical human liver organoid isolation medium 
(advanced DMEM/F12 supplemented with 1\% penicillin/streptomycin, 1\% GlutaMAX, 10 mM HEPES, 1:50 B27 supplement (without vitamin A), 1:100 N2 supplement, $1.25 \mathrm{mM} \mathrm{N}$-acetyl-L-cysteine, 10\% (vol/vol) Rspo-1 conditioned medium, 30\% (vol/vol) Wnt3a-conditioned medium, $10 \mathrm{mM}$ nicotinamide, $10 \mathrm{nM}$ (Leu15)-gastrin I, $50 \mathrm{ng} / \mathrm{ml} \mathrm{EGF,} 100 \mathrm{ng} / \mathrm{ml} \mathrm{FGF,} 25 \mathrm{ng} / \mathrm{ml} \mathrm{HGF,} 10 \mu \mathrm{M}$ forskolin, $5 \mu \mathrm{M}$ A8301, $25 \mathrm{ng} / \mathrm{ml}$ Noggin and $10 \mu \mathrm{M}$ Y27632). The plates were transferred to humidified $37^{\circ} \mathrm{C} / 5 \% \mathrm{CO}_{2}$ incubators containing either $2 \% \mathrm{O}_{2}$ or at ambient $\mathrm{O}_{2}$. The medium was changed every 4 days, and organoids were passaged every $1-4$ weeks.

\section{Primary human liver cancer samples}

Primary human liver cancer and adjacent tissues were obtained from HCC patients at the Cancer Hospital, Chinese Academy of Medical Sciences. Informed consent was obtained from all participants, in accordance with the Declaration of Helsinki. The study was approved by the Ethics Committee of the Cancer Hospital, Chinese Academy of Medical Sciences. All participants provided written informed consent to publish information that identifies individuals. Our study is compliant with the 'Guidance of the Ministry of Science and Technology (MOST) for the Review and Approval of Human Genetic Resources', which requires formal approval for the export of human genetic material or data from China.

\section{Cell culture}

HepG2, Huh7, Hep3B, HCCLM3, Hepa1-6 and H22 cells were purchased from the China Center for Type Culture Collection (CCTCC). HEK 293T cells were purchased from Shanghai Bioleaf Biotech Co. Ltd. The cells had recently been authenticated by short tandem repeat (STR) profiling and characterized by mycoplasma detection and cell viability detection. HepG2 cells were cultured and maintained in MEM (Invitrogen, 11090081) supplemented with 10\% fetal bovine serum (FBS; Invitrogen, CA, USA) and nonessential amino acids (Invitrogen, 11140050) under 5\% carbon dioxide. HCCLM3, Huh7, Hepa1-6 and HEK 293 T cells were cultured and maintained in DMEM supplemented with $10 \%$ FBS. Hep3B cells were cultured and maintained in MEM-EBSS supplemented with $10 \%$ FBS. H22 cells were cultured and maintained in RPMI 1640 medium supplemented with $10 \%$ FBS.

\section{Generation of stable cell lines}

To generate cells with stable SCARB2 knockout, SCARB2 ${ }^{\text {Cas9-1 }}$ and SCARB2 ${ }^{\text {Cas9-2 }}$ lentiviral particles were purchased from TransOMIC Technologies Inc. and were then transfected into HepG2 or HCCLM3 cells. The gRNA sequences targeting SCARB2 were gRNA1: GTGTAGACCAGAGTATCGAGAG and gRNA2: GGTGACCAGCGTCACGCTGCG. After $24 \mathrm{hr}$ of infection, stable cells were selected in medium containing $2.5 \mu \mathrm{g} / \mathrm{ml}$ or $4 \mu \mathrm{g} / \mathrm{ml}$ puromycin (Gibco, CA, USA) for 14 days. After 2-3 passages in the presence of puromycin, cultured cells were used for experiments without cloning.

\section{Generation of spontaneous HCC model Mice}

Hepatocarcinogenesis was induced by DEN as previously described. In brief, 14-day-old male Scarb2 ${ }^{C r e E R T 2-E G F P}$ mice were injected intraperitoneally with DEN ( $25 \mathrm{mg} / \mathrm{kg}$ body weight). Mice were euthanized via $\mathrm{CO}_{2}$ inhalation 8 months after DEN injection. Cre ${ }^{A l b} M y c$ mice with hepatocyte-specific $M y c$ overexpression were generated by mating $M y c^{F / F}$ mice with $C r e^{A l b}$ mice. $C r e^{A l b} M y c$ mice spontaneously developed liver cancer at approximately 2 months old.

To determine the role of Scarb2 in hepatocarcinogenesis, Cre ${ }^{A l b} S c a r b 2^{F / F}$ or $C r e^{A l b} S c a r b 2^{F /+}$ mice with hepatocyte-specific Scarb2 deletion were first generated by mating $S c a r b 2^{F / F}$ mice with $C r e^{A l b}$ mice. $M y c^{\mathrm{F} / \mathrm{F}}$ mice were crossed with $S c a r b 2^{\mathrm{F} / \mathrm{F}}$ mice to generate $S c a r b 2^{\mathrm{F} / \mathrm{F}} M y c$ and $S c a r b 2^{\mathrm{F} /+} M y c$ mice, which were then crossed with $C r e^{A l b} S c a r b 2^{F /+}$ mice to generate $C r e^{A l b} S c a r b 2^{F /+} M y c$ and $C r e^{A l b} S c a r b 2^{F / F} M y c$ trigenic mice. These mice were monitored for tumor incidence for 4 months.

To induce overexpression of Myc in Scarb2 CreERT2-EGFP mice, Scarb2 CreERT2-EGFP $R 26^{\text {Tomato }}$ mice were crossed with Myc ${ }^{\mathrm{F} / \mathrm{F}}$ mice to generate

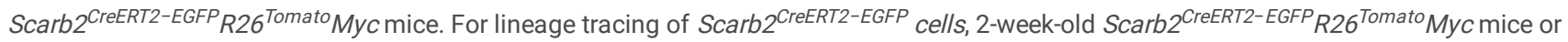
Scarb2 ${ }^{\text {CreERT2-EGFP }}{ }^{2} 6^{\text {Tomato }}$ mice were induced by intraperitoneal injection of $1.5 \mathrm{mg}(150 \mu \mathrm{l} \mathrm{of} 10 \mathrm{mg} / \mathrm{ml})$ tamoxifen (Sigma-Aldrich, 06734) diluted in corn oil (Sigma) for 3 consecutive days.

\section{Establishment of PDX animal models}

Fresh HCC tissue was fragmented into small pieces $\left(1-3 \mathrm{~mm}^{3}\right)$ in medium. The tissue fragments were diluted with Matrigel (Corning, 354248) at a 1:1 ratio and mixed well by shaking. After NSG mice were shaved and anesthetized, the tissue mixture was implanted subcutaneously into these mice. Early passages (1-5) of primary tumor tissues from these PDX models were performed according to the above method.

\section{scRNA-seq assay}

In scRNA-seq assay, human primary HCC cells isolated from tumor tissues of PDX mice were counted three times by three individuals independently and 15,270 live cells were finally used for 10x Genomics. The scRNA-Seq libraries were prepared with single cell 3' Library and Gel Bead Kit V3 (10x Genomics, 1000075), the cell suspension (300-600 living cells per microliter determined by Count Star) was loaded onto the Chromium single cell controller (10x Genomics) to generate single-cell gel beads in the emulsion according to the manufacturer's protocol. In short, single cells were suspended in PBS containing $0.04 \%$ BSA. Reverse transcription was performed on a S1000TM Touch Thermal Cycler (Bio Rad) at $53^{\circ} \mathrm{C}$ for 45 min, followed by $85^{\circ} \mathrm{C}$ for 5 min, and hold at $4^{\circ} \mathrm{C}$. The cDNA was generated and then amplified, and quality assessed using an Agilent 4200 (performed by CapitalBio Technology, Beijing). The libraries were finally sequenced using an Illumina Novaseq6000 sequencer with a sequencing depth of at least 100,000 reads per cell with pair-end 150 bp (PE150) reading strategy (performed by CapitalBio Technology, Beijing). The Cell Ranger Software Suite (Version 3.0.2) was used to perform sample de-multiplexing, barcode processing, and single-cell 3' UMI counting with human GRCh38 as the reference genome. The filtered gene-barcode matrix was analyzed by PCA. Then Uniform Manifold Approximation and Projection (UMAP) was performed on the top 50 principal components for visualizing the cells.

Plasmid construction 
Human MYC-Flag-tag (HG11346-CF), SCARB2-Flag-tag (HG11063-CF), SCARB2-Myc-tag (HG11063-CM), MAX-His-tag (HG12885-CH), HDAC3-HA-tag (HG11511-CY), and SIRT2-HA-tag (HG10830-NY) plasmids were purchased from Sino Biological Inc. (Beijing, China). The MYC truncations MYC-N (amino acids 1-144), MYC-C (amino acids 360-454), and the central region (amino acids 144-360) were inserted into the pEGFP-C1 vector by standard subcloning techniques. The SCARB2 truncations Scarb2-N (amino acids 1-433), and Scarb2-luminal (amino acids 30-433) were inserted into the pcDNA3.1-myc-his vector (Invitrogen, V85520) by standard subcloning techniques.

\section{Luciferase reporter assay for MYC transcriptional activity}

Cells were seeded in 12-well plates and transfected with E-box-dependent luciferase reporters (Yeasen Inc.) using VigoFect (Vigorous Biotechnology Beijing, China). pTK-Renilla was used as the internal control. Luciferase activity was measured $20-24 \mathrm{hr}$ after transfection using a Dual-luciferase Reporter Assay System (Promega, USA).

\section{Single tumor cell preparation and sphere-forming culture}

Human primary cells were separated with a Tumor Cell Isolation Kit, Human (Miltenyi Biotec, \#130-095-929). The enzyme mix (2.2 ml of DMEM, $100 \mu$ l of Enzyme $\mathrm{H}, 50 \mu \mathrm{l}$ of Enzyme R, $12.5 \mu \mathrm{l}$ of Enzyme A) and 0.05- $0.2 \mathrm{~g}$ of tumor samples with the fat, fibrous areas and necrotic areas removed were prepared. The tumor was cut into small pieces of 2-4 mm. The tissue pieces were transferred into a gentleMACS C tube containing the enzyme mix. Dissociation was initiated by running the gentleMACS program 37C_h_TDK_3. The sample was resuspended, and the cell suspension was filtered through a MACS SmartStrainer $(40 \mu \mathrm{m}$ or $70 \mu \mathrm{m})$ placed on a $50 \mathrm{ml}$ tube. The cells and MACS SmartStrainer $(40 \mu \mathrm{m}$ or $70 \mu \mathrm{m})$ were washed with $20 \mathrm{ml}$ of DMEM. The mouse primary cells were separated with a Tumor Cell Isolation Kit, Mouse (Miltenyi Biotec, 130-110-187). The enzyme mix (2.35 ml of DMEM, $100 \mu$ l of Enzyme D, 50 $\mu \mathrm{l}$ of Enzyme R, $12.5 \mu \mathrm{l}$ of Enzyme A) and $0.04-1 \mathrm{~g}$ of tumor samples with the fat, fibrous areas and necrotic areas removed were prepared. The next steps were the same as described above. The cell suspension was centrifuged at $300 \mathrm{~g}$ for $7 \mathrm{~min}$ and resuspended in DMEM/F12.

Cells were suspended in serum-free advanced DMEM/F12 supplemented with $100 \mathrm{IU} / \mathrm{ml}$ penicillin, $100 \mu \mathrm{g} / \mathrm{ml}$ streptomycin, $20 \mathrm{ng} / \mathrm{ml}$ human recombinant epidermal growth factor (hrEGF), $20 \mathrm{ng} / \mathrm{ml}$ human recombinant basic fibroblast growth factor (hrbFGF), 1\% nonessential amino acids, $1 \%$ GlutaMAX, $2 \%$ B27 supplement (Invitrogen, USA), and 1\% N2 supplement (Invitrogen, Carlsbad, CA, USA). Cells were subsequently cultured in ultra-low attachment 6-well plates (Corning Inc., Corning, NY, USA) at a density of no more than 5,000 cells/well.

\section{LDA}

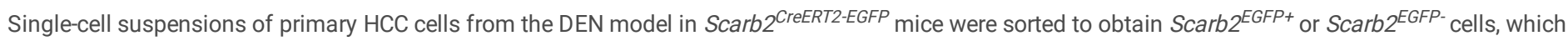
were then injected into BALB/c nude mice. Single-cell suspensions of primary HCC cells from the spontaneous $\mathrm{HCC}$ model in $\mathrm{Cre}^{\mathrm{Alb}} \mathrm{Myc}, \mathrm{Cr} \mathrm{e}^{\mathrm{Alb}} \mathrm{Scarb} 2^{\mathrm{F} /+} \mathrm{Myc}$ or $C r e^{A l b} S c a r b 2^{F / F} M y c$ mice were sorted to obtain HCC cells, which were then injected into BALB/c nude mice. Single-cell suspensions of HCC cells from subcutaneous HCC tumors were sorted to obtain HCC cells, which were then injected into BALB/c nude mice. The outgrowths were analyzed at 8 weeks post transplantation. The tumorigenicity of the transplanted cell suspension was calculated using an extreme limiting dilution assay (ELDA).

\section{Mouse models of tumor growth and metastasis}

To evaluate the effect of combined sorafenib treatment and SCARB2 knockout on the inhibition of tumor growth and metastasis, BALB/c nude mice (5 to 6 weeks old, male) were earmarked before grouping and were then randomly separated into four groups ( $\mathrm{n}=8$ per group) in a blinded manner by an independent person. The tumor growth model in BALB/c-nude mice was established by subcutaneous (s.c.) injection of $2 \times 10^{6} \mathrm{HepG} 2$ cells or HepG2 SCARB2 ${ }^{\text {cas } 9}$ cells diluted with $100 \mu \mathrm{l}$ of Matrigel (Corning, 354230) at a 1:1 ratio. Tumor growth was monitored, and the tumor size was measured using calipers. To establish the mouse model of experimental metastasis, BALB/C-nude mice were injected via the tail vein with $2 \times 10^{6} \mathrm{HCCLM} 3$ cells or HCCLM3 $S C A R B 2^{\text {Cas9 }}$ cells. To evaluate the survival rate, these mice were monitored for more than 3 months. The number of lung metastases was determined.

\section{Immunoprecipitation, immunoblotting, immunostaining and HCC tissue microarray analysis}

Co-IP was performed as described previously. Cells were harvested and lysed for 30 min in co-IP buffer supplemented with a complete protease inhibitor (Cell Signaling Technology, 5817), 1 mM trichostatin A (Selleck, S1045), and 5 mM nicotinamide (MedChemExpress, HYB0150) on ice. Centrifugation was performed to obtain the supernatant, which was then incubated first with the indicated antibodies at $4^{\circ} \mathrm{C}$ overnight, and then with Protein A/G Plus-Agarose (Santa Cruz Biotechnology, TX, USA) at $4^{\circ} \mathrm{C}$ for $2 \mathrm{hr}$. Soluble lysates were incubated with the indicated anti-Myc magnetic beads (Bimake.com, B26302), antiFlag affinity gel (Bimake.com, B23102) or anti-HA affinity gel (Bimake.com, B23302) at $4^{\circ} \mathrm{C}$ overnight. Complexes were eluted from the beads and were then boiled for $10 \mathrm{~min}$. The precipitated proteins were subjected to SDS-PAGE and immunoblotting with the corresponding antibodies. For immunoblot analysis, proteins were extracted from cells and liver tissues using RIPA buffer (Cell Signaling Technology, MA, USA). The protein concentrations were determined with a BCA Protein Assay Kit. Protein extracts were separated by SDS-PAGE, transferred onto PVDF membranes, and subjected to immunoblot analysis. Images of the Western blots were acquired with a Tanon 5200 chemiluminescent imaging system (Tanon, Shanghai, Beijing).

For immunofluorescence ${ }^{42}$ and colocalization assays, cells were seeded in 12-well plates and processed differently according to the experimental requirements. Next, the cells were briefly washed with PBS and fixed with $3.7 \%$ formaldehyde in PBS for 10 min, washed three times with PBS and permeabilized with $0.5 \%$ Triton X-100 for $15 \mathrm{~min}$. Then, the cells were washed with PBS three times and blocked with $3 \%$ bovine serum albumin (BSA) for $1 \mathrm{hr}$ 
at $37^{\circ} \mathrm{C}$. Samples were incubated with primary antibodies overnight at $4^{\circ} \mathrm{C}$ and with secondary antibodies for $2 \mathrm{hr}$. Nuclei were stained with 4',6-diamidino-2phenylindole (DAPI) in blocking buffer. Images were acquired using a confocal microscope (Olympus Microsystems, CA, USA). Quantitative image analysis was performed with Imaris 9.3.1 software. The Pearson correlation coefficient was used to analyze colocalization between two target proteins.

For immunohistochemical analysis, tissue sections were deparaffinized in xylene and rehydrated through a graded alcohol series and distilled water. Antigen retrieval was carried out in a microwave with citrate buffer ( $10 \mathrm{mM}$ sodium citrate buffer, $\mathrm{pH} 6.0$ ) at a subboiling temperature for 15 min. Sections were permeabilized with $0.5 \%$ Triton X-100 in PBS for 20 min. Endogenous peroxidase activity was blocked with $3 \% \mathrm{H}_{2} \mathrm{O}_{2}$ solution for 10 min, and the sections were then washed three times with PBS. Blocking buffer (3\% BSA/PBS) was added to the sections and incubated for 30 min. The sections were then incubated with the indicated primary antibodies at $4^{\circ} \mathrm{C}$ overnight. After washing three times, the sections were incubated for 30 min with the corresponding secondary antibodies at room temperature. Signals were detected with freshly made DAB substrate solution (ZSGB-BIO Company, Beijing, China). The sections were then counterstained with hematoxylin, dehydrated, and mounted with coverslips. Images were acquired using an Olympus DP72 microscope (Olympus Microsystems, CA, USA) and analyzed with Image-Pro Plus 5.1.

For immunoblotting, the following antibodies were used: anti-GAPDH (ZSGB-BIO TA-08, 1:2000), anti-MYC (D3N8F) (CST, \#13987S, 1:1000), anti-MYC (CST, \#9402S, 1:1000), anti-Max (S20) (CST, \#4739, 1:1000), anti-acetylated lysine (CST, \#9441, 1:1000), anti-HDAC3 (7G6C5) (CST, \#3949, 1:1000), anti-SCARB2 (Abcam, ab176317, 1:1000), anti-phospho-MYC (Thr58) (E4Z2K) (CST, \#46650, 1:1000), anti-phospho-MYC (Ser62) (E1J4K) (CST, \#13748, 1:1000), anti-GCN5 (Abcam, ab217876, 1:1000), anti-p44/42 MAPK (Erk1/2) (CST, \#4695, 1:1000), anti-CDK7 (CST, \#2090, 1:1000), anti-BRD4 (CST, \#13440, 1:1000), anti-KAT5 (Abcam, ab151432, 1:1000), anti-Cyclin A2 (E1D9T) (CST, \#91500S, 1:1000), anti-Cyclin D (CST, \#2922, 1:1000), anti-Cyclin E1 (D7T3U) (CST, \#20808, 1:1000), anti-p300 (D8Z4E) (CST, \#86377, 1:1000), anti-CDK4 (D9G3E) (CST, \#12790, 1:1000), anti- N-Cadherin (D4R1H) (CST, \#13116, 1:1000), anti-elF3A (D51F4) (CST, \#3411, 1:1000), anti-elF4E (CST, \#9742, 1:1000), anti-IRP2 (D6E6W) (CST, \#37135, 1:1000), anti-phospho-IRS-1 (Ser1101) (CST, \#2385, 1:1000), antiPKM1/2 (CST, \#3186, 1:1000), anti-Myc-tag (MBL, \#562, 1:1000), anti-GFP-tag (MBL, \#598, 1:1000), anti-DDDDK-tag (MBL, PM020, 1:1000), anti-HA-tag (MBL, \#561, 1:1000), and anti-His-tag (MBL, PM032, 1:1000). For immunofluorescence and immunohistochemistry, the following antibodies were used: anti-MYC (R\&D, AF3696, 1:100), anti-MYC (R\&D, MAB3696, 1:100), anti-MYC (Novus, NB600-302, 1:100), anti-SCARB2 (Thermo Fisher, PA5-20540, 1:100), anti-HDAC3 (Novus, NB500-126, 1:100), anti-GFP (Abcam, ab13970, 1:100), anti-RFP (Abcam, ab62341, 1:100), anti-HNF-4-alpha (Abcam, ab199431, 1:100), anti-Albumin (Abcam, ab207327, 1:100), Alexa Fluor 488 (Thermo Fisher, R37114, 1:200), Alexa Fluor 488 (Thermo Fisher, R37118, 1:200), Alexa Fluor 488 (Abcam, ab150173), Alexa Fluor 555 (Thermo Fisher, A-31572,1:200), Alexa Fluor 555 (Thermo Fisher, A-31570, 1:200), Alexa Fluor 555 (Thermo Fisher, A-21432, 1:200), Alexa Fluor 647 (Thermo Fisher, A-31571, 1:200), and Alexa Fluor 647 (Thermo Fisher, A-31573, 1:200). For ChIP-seq, the following antibody was used: anti-MYC (D3N8F) (CST, \#13987S, $10 \mu \mathrm{g} / \mathrm{ChIP})$.

\section{Flow cytometry}

Primary cell suspensions from liver tissue with or without Scarb2 deletion were directly labeled. Fluorescently labeled antibodies against the following surface proteins were used for mouse cell staining: APC anti-mouse F4/80 (Cat. 123116, BioLegend), APC/Cyanine7 anti-mouse CD45 (Cat. 103116, BioLegend), PE anti-mouse CD31 (Cat. 102407, BioLegend), Percp/Cyanine5.5 anti-mouse CD68 (Cat. 137010, BioLegend), PE anti-mouse CD24 (101807, Biolegend), PE/Cyanine7 anti-mouse CD133 (Cat. 141209, BioLegend) and APC anti-mouse Ep-CAM (Cat. 118214, BioLegend). Then, cells were washed, and data were acquired using a FACSCanto II flow cytometer ${ }^{40}$ and analyzed with FCS EXPRESS software.

\section{Cell Counting Kit-8 (CCK-8) assay}

For the CCK-8 assay, HepG2 and HCCLM3 cells with or without SCARB2 deletion were seeded at a density of 1000 cells/well in 96 -well plates. Cells were cultured for $1,2,3,4$ or 5 days. To generate the time effect curve of sorafenib treatment, $C T R L^{\text {cas } 9}$ and $S C A R B 2^{\text {cas } 9}$ cells were seeded in 96 -well plates at a

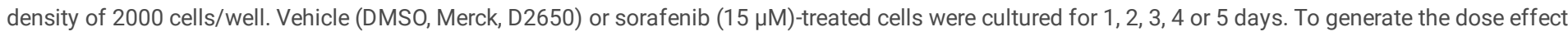
curve of sorafenib treatment, vehicle (DMSO)- or sorafenib $(2,4,8,16,32,62.5,125,250,500 \mu \mathrm{M})$-treated cells were cultured for $24 \mathrm{hr}$. To evaluate the antiproliferative effects of combined treatment with PMB and sorafenib, HepG2, H22, Hepa 1-6, Huh7, Hep3B and HCCLM3 cells were seeded in 96-well plates at a density of 10000 cells/well. Cells were treated with sorafenib $(15 \mu \mathrm{M})$ and an appropriate concentration of inhibitor and cultured for $24 \mathrm{hr}$. Subsequently, $10 \mu \mathrm{l}$ of CCK-8 solution (Dojindo, CK04) was added to each well, and the plates were incubated at $37^{\circ} \mathrm{C}$ for $2 \mathrm{hr}$. Finally, the absorbance was measured at 450 $\mathrm{nm}$.

\section{Invasion assay}

Transwell assays were performed using Millicell inserts ( $8.0 \mu \mathrm{m}$, Millipore, Billerica, MA, USA) to evaluate cell invasion. Millicell inserts were precoated with 10 $\mu \mathrm{g} / \mathrm{ml}$ fibronectin and Matrigel (1:8; BD Bioscience, Bedford, MA, USA) and then allowed to dry. Millicell inserts were placed into the wells of a 24-well plate containing culture medium supplemented with $10 \%$ FBS. Cells $\left(5 \times 10^{4}\right.$ cells/well) were starved overnight and were then seeded in the upper chambers without FBS culture medium. 12 hours later, the migrated cells were fixed with paraformaldehyde, stained with $0.1 \%$ crystal violet and counted.

\section{Real-time PCR and RNA interference}

Total RNA was extracted using TRIzol (Invitrogen, CA, USA) according to the manufacturer's instructions. Total cellular RNA was reverse transcribed using oligo(dT) primers and M-MLV reverse transcriptase (Transgen Biotech, Beijing, China). PCR was performed using a MyCycler thermal cycler and analyzed 
using LineGene 9600. The PCR primer sequences were as follows: Gapdh forward, 5'-CATCACTGCCACCCAGAAGACTG-3'; Gapdh reverse, 5'-

ATGCCAGTGAGCTTCCCGTTCAG-3'; Tfap4 forward, 5'-GACGCGAGATTGCCAACAGCAA-3'; Tfap4 reverse, 5'-TGCTGTCTGCTGGAGAATGGCT-3'; Pld6 forward, 5'TCTGCCTCTTCGCCTTCTCCAG-3'; Pld6 reverse, 5'-GTAGTCGCAGTCAGTGATGACC-3'; Glut4 forward, 5'-GGTGTGGTCAATACGGTCTTCAC-3'; Glut4 reverse, 5'AGCAGAGCCACGGTCATCAAGA-3'; Glut1 forward, 5'-GCTTCTCCAACTGGACCTCAAAC-3'; Glut1 reverse, 5'-ACGAGGAGCACCGTGAAGATGA-3'; Cdk4 forward, 5'CATACCTGGACAAAGCACCTCC-3'; Cdk4 reverse, 5'-GAATGTTCTCTGGCTTCAGGTCC-3'; Ccne 1 forward, 5'-AAGCCCTCTGACCATTGTGTCC-3'; Ccne 1 reverse, 5'CTAAGCAGCCAACATCCAGGAC-3'; Ccnd 1 forward, 5'-GCAGAAGGAGATTGTGCCATCC-3'; Ccnd 1 reverse, 5'-AGGAAGCGGTCCAGGTAGTTCA-3'; Ccna 1 forward, 5'-GCTACTGAGGATGGAGCATCTG-3'; and Ccna 1 reverse, 5'-CAGCTTCCAGAAGGCTCAGTTC-3'. TIP60 siRNA (sc-37967), GCN5 siRNA (sc-37947), and p300 siRNA (sc-29432) were purchased from Santa Cruz Biotechnology.

\section{ChlP-seq}

ChIP assays were performed according to the manufacturer's protocol using a SimpleChIP®Plus Sonication Chromatin IP Kit (Cell Signaling Technology, Danvers, MA, USA, \#56383). In brief, HCCLM3 cells were fixated with 1\% formaldehyde (Sigma-Aldrich, F8775) for 8 min, incubated with glycine (50 mM final concentration) for $10 \mathrm{~min}$ and washed three times with PBS. After cell lysis and chromatin extraction, chromatin was sonicated into fragments of $100-500$ bp using a BioRuptor sonicator (Diagenode) and was then centrifuged at $16,000 \times \mathrm{g}$ for $10 \mathrm{~min}$ at $4^{\circ} \mathrm{C}$. To control for experimental variation, our spike-in data were normalized to a spike-in control consisting of a small amount of chromatin from another species. In brief, $\mathrm{H} 22$ cell lysates were sonicated exactly as described above and added to HCCLM3 cells at a 1:100 ratio. The spiked lysates were incubated overnight at $4^{\circ} \mathrm{C}$ with a ChIP-grade antibody specific for MYC (CST, \#13987S, $10 \mathrm{mg} / \mathrm{ChIP}$ ), which was coupled to magnetic beads. The precipitated material was eluted (input chromatin was used as a control), crosslinking was reversed, and DNA was purified by chloroform/phenol extraction and resuspended in DNA elution buffer. ChIP-seq libraries were generated by sequential DNA fragmentation, end repair, dA tailing, adaptor ligation and PCR amplification. A Qubit® 3.0 A fluorometer was used for quantitation of the ChIP library. An Agilent 2100 Bioanalyzer was used to determine the insert sizes of the library. A StepOnePlusTM Real-Time PCR system was used to assess the molality of the library, which was required to exceed $10 \mathrm{mM}$ for sequencing.

\section{ChIP-seq data analysis}

ChIP libraries were sequenced on the Illumina HiSeq platform with 150 bp paired-end reads. After quality control, the clean reads were aligned to the human reference genome (Homo sapiens GRCh38.87v2) with Bowtie v2.1.0. Unambiguously mapped reads were retained for subsequent generation of binding profiles, heatmap visualization and peak calling. Model-based Analysis of ChIP-seq (MACS2) version 2 was used to identify regions in ChIP samples in which the signal was enriched over the background signal from the corresponding input sample, and a $P$ value of $10^{\wedge}-9$ was used as the cutoff to identify statistically significant peaks. Mapped reads were visualized using Integrative Genomics Viewer (IGV). We next used the murine spike-in to quantitatively normalize different samples. In brief, the total number of reads aligned to the mouse $\mathrm{mm} 9$ genome assembly was used as a normalization factor to scale ChIP-seq data sets produced from equal numbers of cells. Bamliquidator (https://github.com/BradnerLab-/pipeline/wiki/bamliquidator, version 1.0) was used to calculate the ChIP-seq read density over a given genomic coordinate. Heatmaps were generated and genome-wide correlation analyses were performed using deepTools2. To visualize density distributions around transcription start sites (TSSs) or ChIP peaks and heatmaps indicating MYC occupancy, plotHeatmap was used. Peaks were annotated using the 'closestBed' tool in the BEDTools suite v2.20.1.

\section{Generation of the MYC K148 mutation with the CRISPR/Cas system}

The sgRNAs for introducing the MYC K148R knock-in mutation were annealed and ligated into the YKO vector. The gRNA sequences targeting MYC K148R were gRNA1: 5'-CAGCTTCTCTGAGACGAGCTTGG-3' and gRNA2: 5'-TTTGCGCGCAGCCTGGTAGGAGG. The repair template designed with a homologous genomic flanking sequence was TCATCATCCAGGACTGTATGTGGAGCGGCTTCTCGGCCGCCGCGAAGCTCGTCTCAGAGAGGCTGGCGTCCTACCAGGCTGCGCGCAAAGACAGCGGCAGCCCGAACC) $1 \mathrm{mg}$ of each sgRNA plasmid was mixed with $1 \mathrm{mg}$ of donor plasmid for transfection into HCCLM3 cells with Lipofectamine 3000 Reagent (Invitrogen, L3000008) according to the manufacturer's instructions. Twelve hours after transfection, HCCLM3 cells were treated with $5 \mathrm{mg} / \mathrm{ml}$ puromycin for $24 \mathrm{hr}$. Then, the puromycin was removed from the cell culture medium, and the cultured cells were sorted into 96 -well plates at 1 cell/well. The cells were incubated and expanded for 2-3 weeks, and all clones were further subjected to genomic DNA extraction (TIANamp Genomic DNA Kit, DP304), PCR amplification of the MYC sequence and Sanger sequencing. The sequences of the PCR primers were as follows: MYC-K148R forward, 5'-CTCGTCTCAGAGAGGCTGGCCTCCT-3' and MYC-K148R reverse, 5'-AGGAGGCCAGCCTCTCTGAGACGAG-3'. The correct K148R knock-in cell clones were selected for further experiments.

\section{Structured Illumination Microscopy (SIM)}

Cells $(20,000)$ were grown on coverslips and fixed with formalin after the indicated treatment. Subsequently, the cells were washed in Tris-buffered saline (TBS) and permeabilized for 5 min with $0.5 \%$ Triton X-100 in TBS. The reaction was quenched for 10 min with $50 \mathrm{nM}$ glycine in TBS, and blocking was performed for $30 \mathrm{~min}$ with $5 \%$ normal mouse serum or normal goat serum in a $0.2 \%$ gelatin-TBS solution depending on the isotype of the secondary antibody. Subsequently, 30- $\mu$ l droplets containing the indicated dilutions of antibodies specific for MYC (R\&D, AF3696, 1:100), SCARB2 (Thermo Fisher, PA5-20540, 1:100) or HDAC3 (Novus, NB500-126, 1:100) were placed on Parafilm in a dark humidified chamber. Coverslips were placed face down on the droplets and incubated overnight at $4^{\circ} \mathrm{C}$. The next day, the coverslips were lifted by adding a small volume $(200 \mu \mathrm{l})$ of TBS under the coverslip. After washing $3 \times 20$ min with $1 \mathrm{ml}$ of $0.2 \%$ gelatin-TBS, the coverslips were incubated with Alexa Fluor 488 (Thermo Fisher, R37114, 1:200) and Alexa Fluor 555 (Thermo Fisher, A$31572,1: 200$ ) secondary antibodies as described for primary antibody incubation for $1 \mathrm{hr}$ at room temperature. After washing $3 \times 10 \mathrm{~min}$ with $1 \mathrm{ml}$ of $0.2 \%$ 
TBS-gelatin and 1 wash with regular TBS, the coverslips were mounted using soft-set mounting medium with DAPI (Vectashield) and sealed with nail polish. Images were acquired using a confocal microscope (Olympus Microsystems, CA, USA) or a GE Healthcare 3D structured illumination microscope. Intensity plots of individual pixels taken from a straight line in the indicated immunofluorescence images were generated by the twin slicer tool in Imaris 3D \& 4D imaging software (Bitplane AG, Switzerland). Images were cropped and processed in Adobe Photoshop. When comparisons were made between images from the same experiment, all levels were adjusted equally, and the ratio between the levels was not altered.

\section{GSEA}

We ranked genes by their association with $C r e^{A l b} S c a r b 2^{F / F} M y c$ mice vs. Cre ${ }^{A l b} M y c$ mice and HepG2 cells vs. PMB treated HepG2 cells using the signal-to-noise metric determined by GSEA according to the log2 fold change values. MYC target gene sets were obtained from a database (http://software.broadinstitute.org/gsea/msigdb/index.jsp).

\section{Mass spectrometry analysis}

Whole-cell lysates of HepG2 cells were immunoprecipitated with an anti-MYC antibody (CST, 9402S) or IgG1 isotype control antibody (CST, 5415) and Protein A/G Plus-Agarose (Santa Cruz Biotechnology) overnight at $4^{\circ} \mathrm{C}$. Interaction complexes were eluted from the beads by heating at $98^{\circ} \mathrm{C}$ for 10 min. Mass spectrometric data analysis was performed by Beijing Qinglian Biotech Co., Ltd.

\section{Surface plasmon resonance analysis}

A BIAcore S200 system (GE Healthcare) was used to analyze the surface plasmon resonance binding kinetics between SCARB2 and the indicated small molecules. In brief, SCARB2 protein (Sino Biological, China) was immobilized onto channel 2 in a CM5 sensor chip (GE Healthcare) through a standard coupling protocol. To measure the binding kinetics, the indicated small molecule (100 0.098 nM in 2-fold serial dilutions) and a buffer blank for baseline subtraction were sequentially injected, with a regeneration step (glycine, $\mathrm{pH} 2.5$ ) performed between each cycle. The equilibrium dissociation constant was calculated with BIA evaluation software.

\section{Mouse models to evaluate tumor growth and combination treatment with sorafenib and PMB}

To evaluate the effect of combination treatment with sorafenib and PMB (polymyxin B sulfate) on tumor growth, BALB/c nude or NSG mice (5 to 6 weeks old, male) were earmarked before grouping and were then randomly separated into four groups ( $n=8$ per group) in a blinded manner by an independent person. To establish the tumor growth model in BALB/c nude mice, $2 \times 10^{6}$ HepG2 or HCCLM3 cells per mouse diluted with $100 \mu$ l of Matrigel (Corning, 354230) at a 1:1 ratio were subcutaneously injected into the right flanks of nude mice. To establish the PDX model, tumor masses $\left(0.1 \mathrm{~cm}^{3}\right)$ were subcutaneously transplanted into NSG mice. Tumors were allowed to grow for 7 days, and mice were then administered vehicle (Kolliphor ${ }^{\circledR}$ HS 15, i.g., once a day), sorafenib (30 mg/kg, i.g., once a day), PMB (25 mg/kg, i.g., once a day), or PMB plus sorafenib for 14 days. During the indicated treatments, tumor burden was evaluated by measuring tumor volumes to determine the therapeutic efficacy in the mouse models and PDX models.

\section{Statistics \& Reproducibility}

No data were excluded from the analyses. For in vivo cancer studies, mice were randomly assigned into experimental groups. The Investigators were not blinded to allocation during experiments and outcome assessment. Unpaired Student's t test in SPSS 13.0 software was used to compare parameters between two groups. One-way analysis of variance (ANOVA) was used to compare parameters among more than two groups. Pearson correlation analysis was used to determine correlations between groups. The Kaplan-Meier method was used to analyze survival. All data are expressed as the mean \pm standard error of the mean (SEM) values. Generally, all experiments were carried out with $\mathrm{n} \geq 3$ biological replicates. All statistical tests were two-tailed. $P<0.05$ was considered statistically significant. All the data presented has been reviewed by a statistician to ensure scientific rigor and diligence of our data. Further information on research design is available in the Nature Research Reporting Summary linked to this article.

\section{Declarations}

\section{Code Availability Statement}

No custom code was used for the analysis. Code will be made available upon reasonable request.

\section{Data availability}

The RNA-seq, scRNA-seq and ChIP-seq data have been uploaded to the NCBI Gene Expression Omnibus (GEO) database under accession numbers GSE185844. All other data supporting the findings of this study are available from the corresponding author upon reasonable request. 
This work was supported by grants from the "Ten thousand plan" - National high-level talents special support plan to K.L., the National Natural Science Foundation of China (8187131307, 82073887 to K.L., 82003798 to F.W.), and the CAMS Innovation Found for Medical Sciences (2016-I2M-1-011 to K.L.).

\section{References}

1. Sung, H. et al. Global Cancer Statistics 2020: GLOBOCAN Estimates of Incidence and Mortality Worldwide for 36 Cancers in 185 Countries. CA Cancer J Clin 71, 209-249, doi:10.3322/caac.21660 (2021).

2. Villanueva, A. Hepatocellular Carcinoma. N Engl J Med 380, 1450-1462, doi:10.1056/NEJMra1713263 (2019).

3. Nio, K., Yamashita, T. \& Kaneko, S. The evolving concept of liver cancer stem cells. Mol Cancer 16, 4, doi:10.1186/s12943-016-0572-9 (2017).

4. Zhou, H. M., Zhang, J. G., Zhang, X. \& Li, Q. Targeting cancer stem cells for reversing therapy resistance: mechanism, signaling, and prospective agents. Signal Transduct Target Ther 6, 62, doi:10.1038/s41392-020-00430-1 (2021).

5. Saygin, C., Matei, D., Majeti, R., Reizes, O. \& Lathia, J. D. Targeting Cancer Stemness in the Clinic: From Hype to Hope. Cell Stem Cell 24, 25-40, doi:10.1016/j.stem.2018.11.017 (2019).

6. Yoshida, M. et al. Sorafenib suppresses extrahepatic metastasis de novo in hepatocellular carcinoma through inhibition of mesenchymal cancer stem cells characterized by the expression of CD90. Sci Rep 7, 11292, doi:10.1038/s41598-017-11848-z (2017).

7. Hu, X. et al. Induction of cancer cell stemness by chemotherapy. Cell Cycle 11, 2691-2698, doi:10.4161/cc.21021 (2012).

8. Hou, Y., Zou, Q., Ge, R., Shen, F. \& Wang, Y. The critical role of CD133(+)CD44(+/high) tumor cells in hematogenous metastasis of liver cancers. Cell Res 22, 259-272, doi:10.1038/cr.2011.139 (2012).

9. Yang, Z. F. et al. Significance of CD90+ cancer stem cells in human liver cancer. Cancer Cell 13, 153-166, doi:10.1016/j.ccr.2008.01.013 (2008).

10. Yamashita, T. et al. EpCAM-positive hepatocellular carcinoma cells are tumor-initiating cells with stem/progenitor cell features. Gastroenterology 136, 1012-1024, doi:10.1053/j.gastro.2008.12.004 (2009).

11. Lee, T. K., Guan, X. Y. \& Ma, S. Cancer stem cells in hepatocellular carcinoma - from origin to clinical implications. Nat Rev Gastroenterol Hepatol, doi:10.1038/s41575-021-00508-3 (2021).

12. Abou-Elella, A., Gramlich, T., Fritsch, C. \& Gansler, T. c-myc amplification in hepatocellular carcinoma predicts unfavorable prognosis. Mod Pathol 9, $95-98$ (1996).

13. Xu, Z. et al. The mTORC2-Akt1 Cascade Is Crucial for c-Myc to Promote Hepatocarcinogenesis in Mice and Humans. Hepatology 70, 1600-1613, doi:10.1002/hep.30697 (2019).

14. Kaposi-Novak, P. et al. Central role of c-Myc during malignant conversion in human hepatocarcinogenesis. Cancer Res 69, 2775-2782, doi:10.1158/00085472.CAN-08-3357 (2009).

15. Holczbauer, A. et al. Modeling pathogenesis of primary liver cancer in lineage-specific mouse cell types. Gastroenterology 145, 221-231, doi:10.1053/j.gastro.2013.03.013 (2013).

16. Faiola, F. et al. Dual regulation of c-Myc by p300 via acetylation-dependent control of Myc protein turnover and coactivation of Myc-induced transcription. Mol Cell Biol 25, 10220-10234, doi:10.1128/MCB.25.23.10220-10234.2005 (2005).

17. Patel, J. H. et al. The c-MYC oncoprotein is a substrate of the acetyltransferases hGCN5/PCAF and TIP60. Mol Cell Biol 24, 10826-10834, doi:10.1128/MCB.24.24.10826-10834.2004 (2004).

18. Febbraio, M., Hajjar, D. P. \& Silverstein, R. L. CD36: a class B scavenger receptor involved in angiogenesis, atherosclerosis, inflammation, and lipid metabolism. J Clin Invest 108, 785-791, doi:10.1172/JCl14006 (2001).

19. Heybrock, S. et al. Lysosomal integral membrane protein-2 (LIMP-2/SCARB2) is involved in lysosomal cholesterol export. Nat Commun 10, 3521, doi:10.1038/s41467-019-11425-0 (2019).

20. Cruz, W. et al. Lipoprotein-Like Nanoparticle Carrying Small Interfering RNA Against Spalt-Like Transcription Factor 4 Effectively Targets Hepatocellular Carcinoma Cells and Decreases Tumor Burden. Hepatol Commun 4, 769-782, doi:10.1002/hep4.1493 (2020).

21. Luo, X. et al. The fatty acid receptor CD36 promotes HCC progression through activating Src/PI3K/AKT axis-dependent aerobic glycolysis. Cell Death Dis 12, 328, doi:10.1038/s41419-021-03596-w (2021).

22. Feng, W. W. et al. CD36-Mediated Metabolic Rewiring of Breast Cancer Cells Promotes Resistance to HER2-Targeted Therapies. Cell Rep 29, 3405-3420 e3405, doi:10.1016/j.celrep.2019.11.008 (2019).

23. Ye, H. et al. Leukemic Stem Cells Evade Chemotherapy by Metabolic Adaptation to an Adipose Tissue Niche. Cell Stem Cell 19, 23-37, doi:10.1016/j.stem.2016.06.001 (2016).

24. Yamayoshi, S. et al. Scavenger receptor B2 is a cellular receptor for enterovirus 71. Nat Med 15, 798-801, doi:10.1038/nm.1992 (2009).

25. Conrad, K. S. et al. Lysosomal integral membrane protein-2 as a phospholipid receptor revealed by biophysical and cellular studies. Nat Commun 8 , 1908 , doi:10.1038/s41467-017-02044-8 (2017).

26. Schmelzer, E. et al. Human hepatic stem cells from fetal and postnatal donors. J Exp Med 204, 1973-1987, doi:10.1084/jem.20061603 (2007).

27. Nebbioso, A. et al. c-Myc Modulation and Acetylation Is a Key HDAC Inhibitor Target in Cancer. Clin Cancer Res 23, 2542-2555, doi:10.1158/10780432.CCR-15-2388 (2017).

28. Wei, X. et al. KAT5 promotes invasion and metastasis through C-MYC stabilization in ATC. Endocr Relat Cancer 26, 141-151, doi:10.1530/ERC-18-0193 (2019). 
29. Menssen, A. et al. The c-MYC oncoprotein, the NAMPT enzyme, the SIRT1-inhibitor DBC1, and the SIRT1 deacetylase form a positive feedback loop. Proc Natl Acad Sci U S A 109, E187-196, doi:10.1073/pnas.1105304109 (2012).

30. Farria, A. T. et al. Transcriptional Activation of MYC-Induced Genes by GCN5 Promotes B-cell Lymphomagenesis. Cancer Res $80,5543-5553$, doi:10.1158/0008-5472.CAN-20-2379 (2020).

31. Poole, C. J. \& van Riggelen, J. MYC-Master Regulator of the Cancer Epigenome and Transcriptome. Genes (Basel) 8, doi:10.3390/genes8050142 (2017).

32. Huch, M. et al. In vitro expansion of single Lgr5+ liver stem cells induced by Wnt-driven regeneration. Nature 494, 247-250, doi:10.1038/nature11826 (2013).

33. Qiu, Q., Hernandez, J. C., Dean, A. M., Rao, P. H. \& Darlington, G. J. CD24-positive cells from normal adult mouse liver are hepatocyte progenitor cells. Stem Cells Dev 20, 2177-2188, doi:10.1089/scd.2010.0352 (2011).

34. Dang, H. T., Budhu, A. \& Wang, X. W. The origin of cancer stem cells. J Hepatol 60, 1304-1305, doi:10.1016/j.jhep.2014.03.001 (2014).

35. Rycaj, K. \& Tang, D. G. Cell-of-Origin of Cancer versus Cancer Stem Cells: Assays and Interpretations. Cancer Res 75, 4003-4011, doi:10.1158/00085472.CAN-15-0798 (2015).

36. Yuan, J., Minter-Dykhouse, K. \& Lou, Z. A c-Myc-SIRT1 feedback loop regulates cell growth and transformation. J Cell Biol 185, 203-211, doi:10.1083/jcb.200809167 (2009).

37. Pei, Y. et al. HDAC and PI3K Antagonists Cooperate to Inhibit Growth of MYC-Driven Medulloblastoma. Cancer Cell 29, 311-323, doi:10.1016/j.ccell.2016.02.011 (2016).

38. Heidelberger, J. B. et al. Proteomic profiling of VCP substrates links VCP to K6-linked ubiquitylation and c-Myc function. EMBO Rep 19, doi:10.15252/embr.201744754 (2018).

39. Kalkat, M. et al. MYC Protein Interactome Profiling Reveals Functionally Distinct Regions that Cooperate to Drive Tumorigenesis. Mol Cell $72,836-848$ e837, doi:10.1016/j.molcel.2018.09.031 (2018).

40. Han, H. et al. Small-Molecule MYC Inhibitors Suppress Tumor Growth and Enhance Immunotherapy. Cancer Cell 36, 483-497 e415, doi:10.1016/j.ccell.2019.10.001 (2019).

41. Li, K. et al. TRIB3 promotes MYC-associated lymphoma development through suppression of UBE3B-mediated MYC degradation. Nat Commun 11, 6316, doi:10.1038/s41467-020-20107-1 (2020).

42. Liang, K. et al. Targeting Processive Transcription Elongation via SEC Disruption for MYC-Induced Cancer Therapy. Cell 175, 766-779 e717, doi:10.1016/j.cell.2018.09.027 (2018).

\section{Figures}




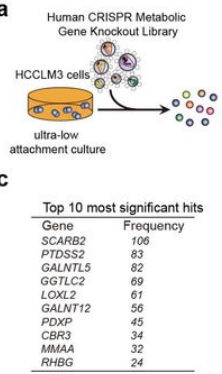

d
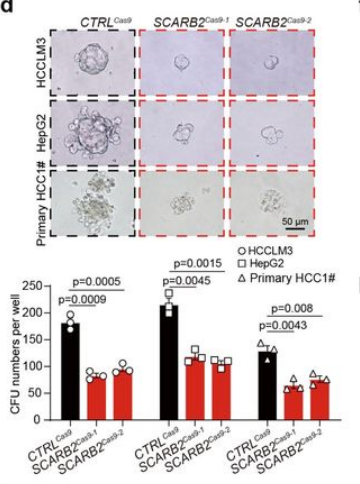

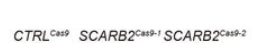

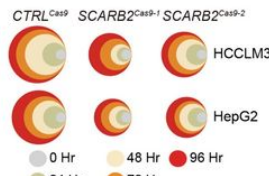

$96 \mathrm{Hr}$

f
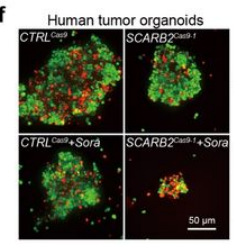

b
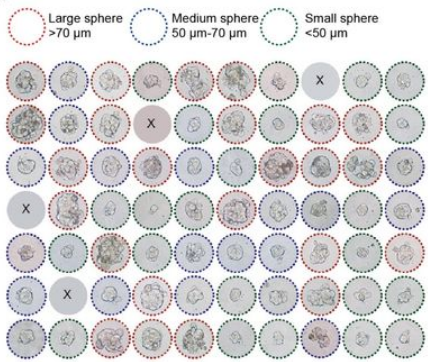

g
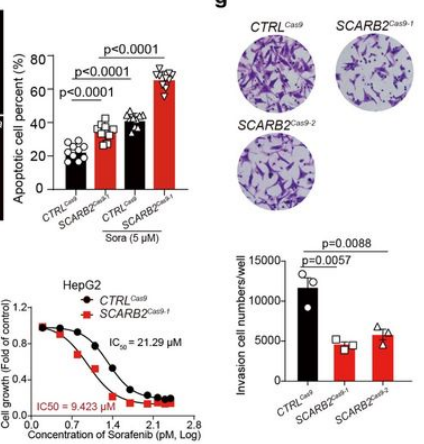

i
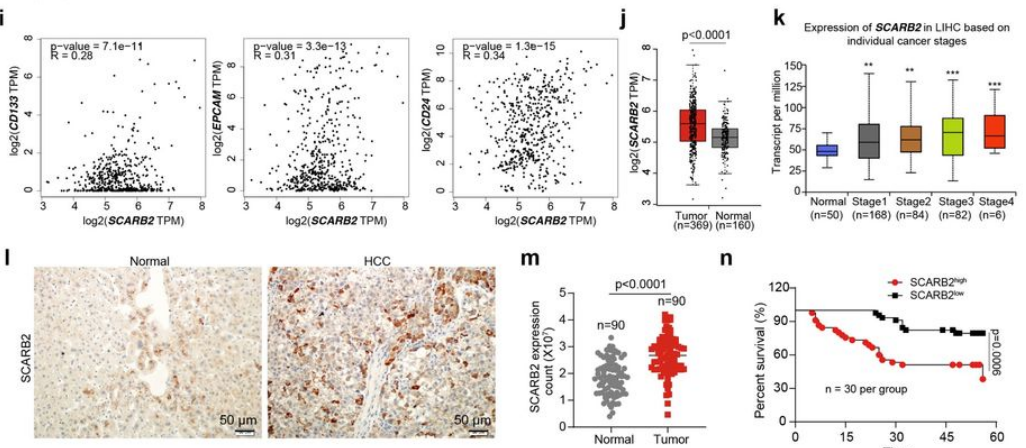

n

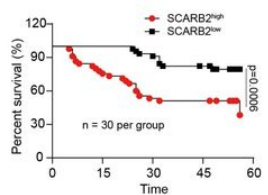

Figure 1

CRISPR library screening identified SCARB2 as a positive modulator of HCC cell stemness.

(a) Schematic diagram illustrating the workflow for human CRISPR metabolic gene knockout library screening. (b) Representative images of tumorspheres of different sizes cultured in ultra-low attachment 96 -well plates. Sphere sizes were classified as follows: large spheres (red circle, $>70 \mu \mathrm{m}$ ), medium spheres (blue circle, $50 \mu \mathrm{m}-70 \mu \mathrm{m}$ ) and smaller spheres (green circle, $<50 \mu \mathrm{m}$ ). (c) Top candidate genes were shown after culture under serum-free and ultra-low attachment conditions. (d) Validation of the effects of SCARB2 knockout on the sphere-forming ability of HCC cells. (e) Relative viability of HCCLM3 and HepG2 cells with or without SCARB2 deletion at the indicated times. Viability was measured by a CCK-8 assay. (f) Immunofluorescence images and quantification of the viability of patient-derived HCC organoids transduced with $C T R L^{C a s 9}$ or $S C A R B 2^{\text {Cas9-1 }}$ in $3 \mathrm{D}$ culture after the indicated treatment. Live and dead cells were identified with calcein acetoxymethyl (calcein-AM) (green) to mark viable cells and ethidium bromide homodimer-1 (red) to mark dead cells.

(g) Representative images and quantification of the invasion assay results for HepG2 cells stably expressing CTRL $^{\text {Cas9 }}$, SCARB2 ${ }^{\text {Cas9-1 }}$, or SCARB2 ${ }^{\text {Cas9-2 }}$. (h)

Effect of SCARB2 depletion on sorafenib sensitivity in HCCLM3 and HepG2 cells. The data are a summary of IC50 values for sorafenib. (i) Pearson's correlation between SCARB2 expression with the known CSC markers (CD133, EPCAM or CD24) using samples from GEPIA database. Transcript per million, TPM. (j) Differential expression of SCARB2 in tumor and healthy liver tissue sample from the GEPIA database. (k) Expression of SCARB2 expression in liver hepatocellular carcinoma (LIHC) based on individual cancer stages. $(\mathbf{l}, \mathrm{m})$ IHC staining of SCARB2 expression in human normal liver and HCC specimens $(n=$ 90). (n) Kaplan-Meier survival curves for patients with HCC stratified by SCARB2 expression were generated. 

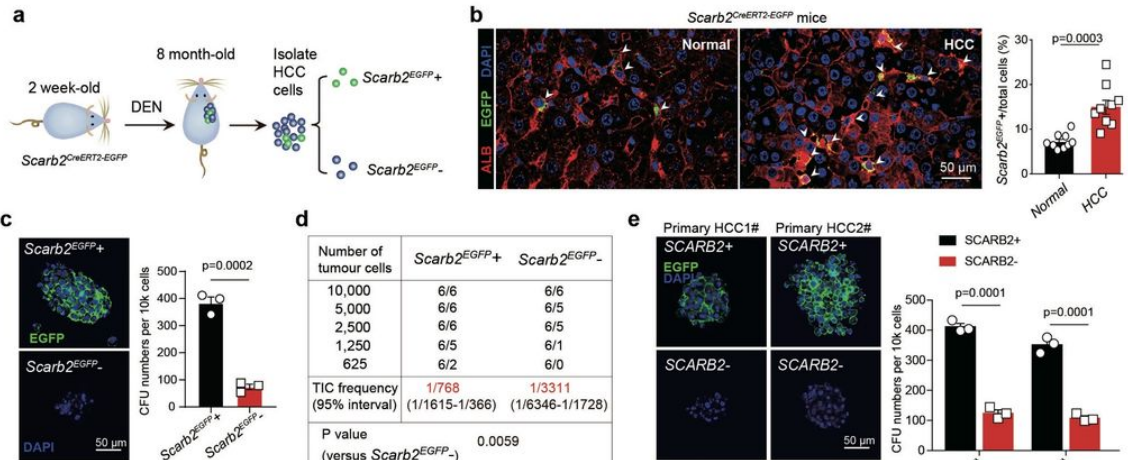

d
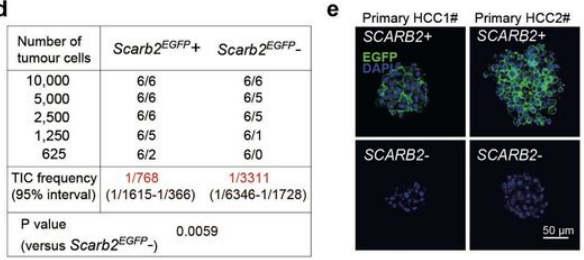

- SCARB2+

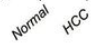

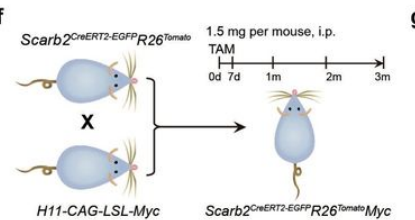

g

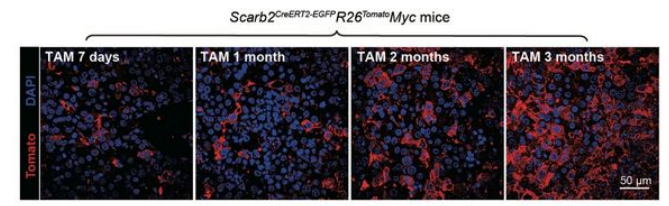

h
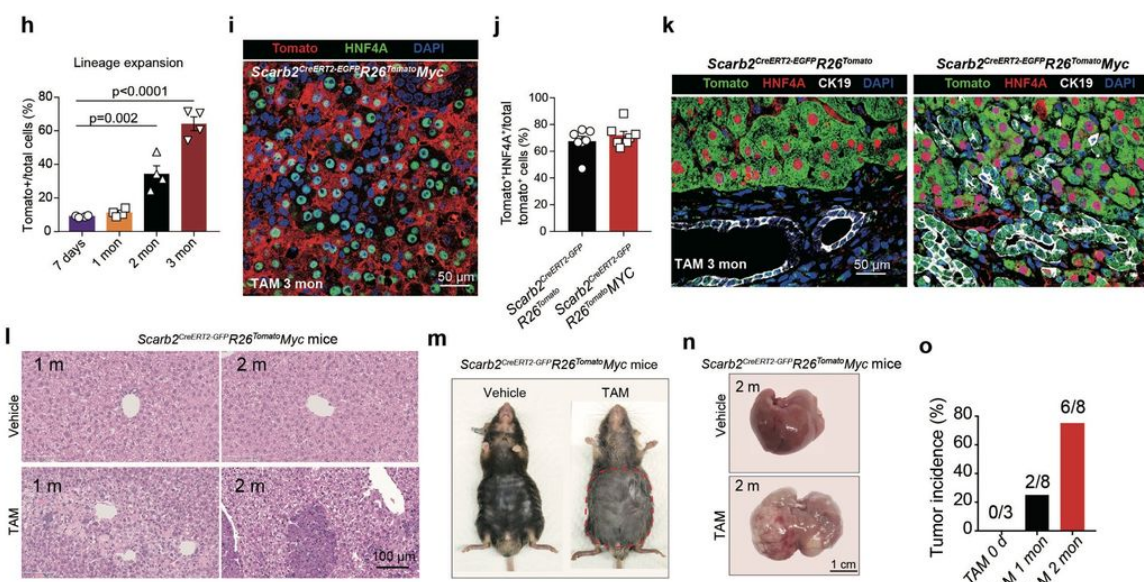

n
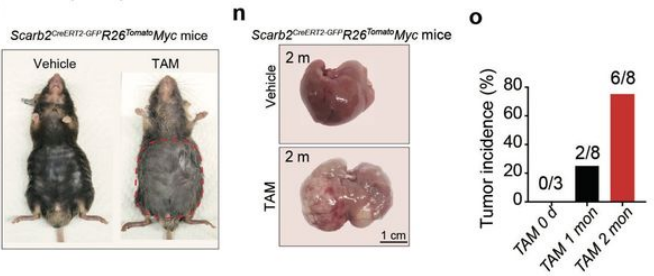

Figure 2

SCARB2+ HCC cells have stem cell-like properties and repopulate HCC tumors.

(a) Schematic diagram of Scarb2 CreERT2-EGFP + HCC cells isolated from Scarb2 ${ }^{\text {CreERT2-EGFP }}$ mice with DEN-induced HCC. (b) Immunofluorescence analysis of

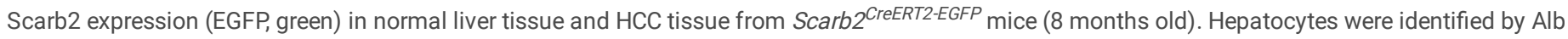
staining (red). (c) Representative images and quantification of tumorspheres formed by Scarb2 ${ }^{\text {EGFP+ }}$ and Scarb2 ${ }^{\text {EGF- }}{ }^{\text {HCC }}$ cells. (d) Tumorigenic cell frequency among Scarb2 ${ }^{\text {EGFP+ }}$ and Scarb2 ${ }^{\text {EGFP- }}$ HCC cells, as analyzed with a LDA. (e) Representative images and quantification of tumorspheres formed by primary SCARB2 ${ }^{+}$and SCARB2 ${ }^{-}$HCC cells isolated from tumor tissues of HCC patients. (f) Schematic diagram of the process for achieving inducible

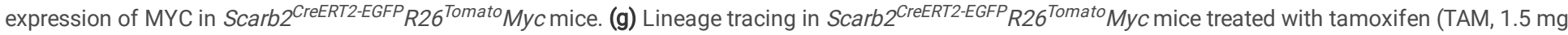
per mouse, 3 consecutive days) and analyzed after the indicated tracing periods by immunofluorescence staining for tomato. (h) Quantification of the tomato+ hepatocyte area in Scarb2 ${ }^{\text {CreERT2-EGFP }}{ }^{2} 26^{\text {Tomato }} \mathrm{Myc}$ mice treated with tamoxifen at the indicated times. (i) Immunofluorescence analysis of tomato (red) and HNF4A expression (green) in HCC tissues of Scarb2 ${ }^{\text {CreERT2-EGFP }}{ }^{26} 6^{\text {Tomato } M y C ~ m i c e ~ t r e a t e d ~ w i t h ~ t a m o x i f e n ~ f o r ~} 3$ months. (j) Quantification of

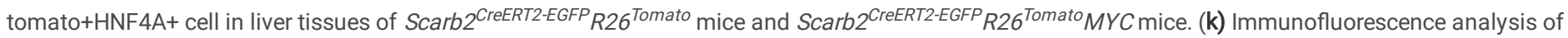
tomato (green), HNF4A (red), and CK19 (grey) expression in liver tissues of Scarb2 ${ }^{\text {CreERT2-EGFP }}{ }^{26} 6^{\text {Tomat }}$ and Scarb2 ${ }^{\text {CreERT2-EGFP }}$ R26 $6^{\text {Tomato }}$ Myc mice treated with tamoxifen for 3 months. (I) Representative H\&E staining of the livers of 1-month-old or 2-month-old Scarb2 ${ }^{\text {CreERT2-EGFP }}{ }^{2} 26^{\text {Tomato }}$ MyC mice treated with

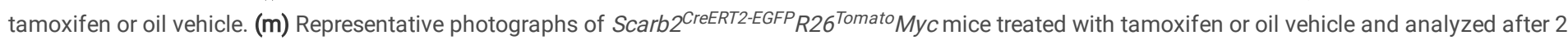
months. (n) Representative images of liver morphology in Scarb2 ${ }^{\text {CreERT2-EGFP }}{ }^{2} 26^{\text {Tomato }}$ Myc mice treated with tamoxifen or oil vehicle and analyzed after the indicated tracing periods. (o) Tumor incidence in Scarb2 ${ }^{\text {CreERT2-EGFP }} R 26^{\text {Tomato }}$ Myc mice treated with tamoxifen after the indicated tracing periods. 
a

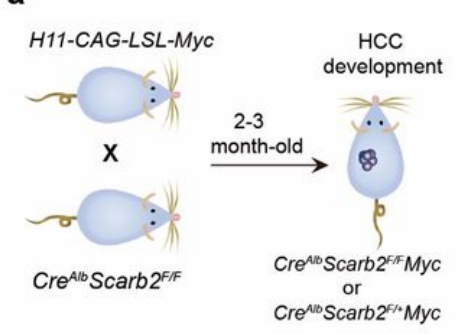

b

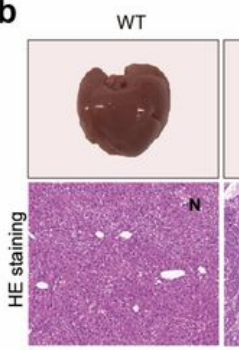

$\mathrm{Cre}^{\mu \mathrm{Mb} M y c}$

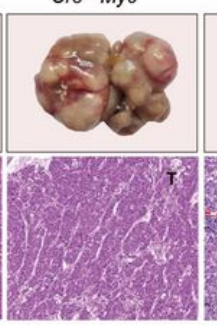

$\mathrm{Cre}^{\mu b} \mathrm{Scarb} 2^{\mathrm{F} / \mathrm{M}} \mathrm{MC}$
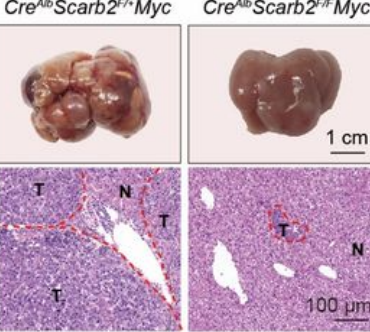

C

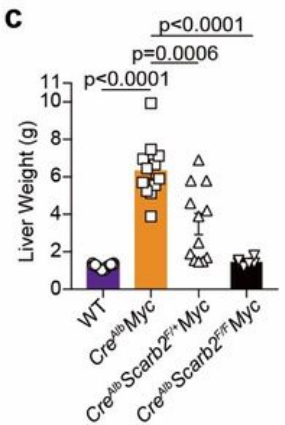

d

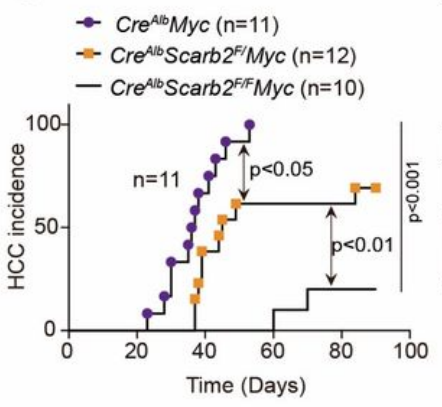

g

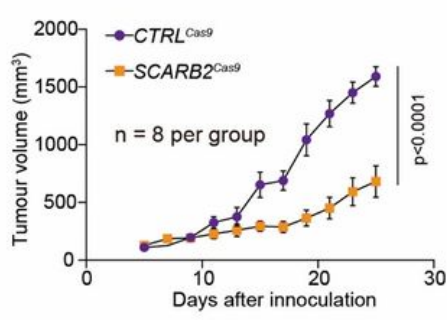

e

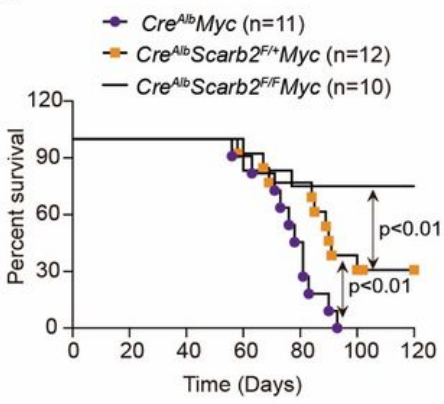

h

CTRL Cas9

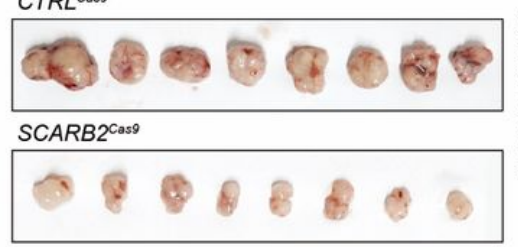

f

\begin{tabular}{|c|c|c|c|}
\hline $\begin{array}{l}\text { Number of } \\
\text { tumour cells }\end{array}$ & $C r e^{A / b} M y c$ & $\begin{array}{l}\text { Cre } e^{A / b} S c a r b 2^{F / 4} \\
\text { Myc }\end{array}$ & 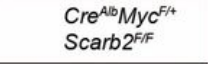 \\
\hline 100,000 & $10 / 10$ & $8 / 10$ & $4 / 10$ \\
\hline 5,0000 & $9 / 10$ & $6 / 10$ & $2 / 10$ \\
\hline 25,000 & $9 / 10$ & $3 / 10$ & $1 / 10$ \\
\hline 12,500 & $6 / 10$ & $3 / 10$ & $1 / 10$ \\
\hline 6,250 & $5 / 10$ & $1 / 10$ & $0 / 10$ \\
\hline $\begin{array}{l}\text { TIC frequency } \\
\text { (95\% interval) }\end{array}$ & $\begin{array}{c}1 / 13506 \\
(1 / 21063-1 / 8660)\end{array}$ & $\begin{array}{c}1 / 56715 \\
(1 / 89509-1 / 35936)\end{array}$ & $\begin{array}{c}1 / 206291 \\
(1 / 413668-1 / 102875)\end{array}$ \\
\hline \multirow{2}{*}{$P$ value } & \multicolumn{2}{|c|}{$p<0.0001$} & \\
\hline & & $p<0.0001$ & \\
\hline
\end{tabular}

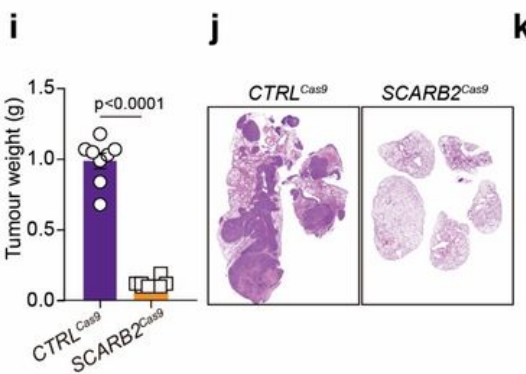

$\mathbf{k}$

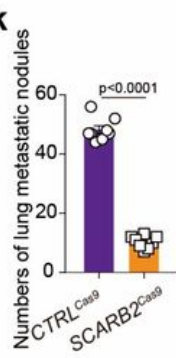

Figure 3

Scarb2 deletion suppresses HCC initiation and progression.

(a) Scheme used to establish the model of spontaneous HCC with targeted MYC knock-in and Scarb2 knockout in the liver. (b) Representative photographs of intrahepatic tumor burden (top) and H\&E staining of tumor tissue (bottom) in the indicated mice 8 weeks after birth. (c) Liver weight in the indicated mice. (d) Incidence of spontaneous HCC in indicated mice. (e) Survival curves for indicated mice. (f) Effects of Scarb2 on the incidence of tumor formation from secondary transplanted HCC cells in indicated mice. (g-i) Growth, photographs, and weights of tumors in nude mice injected subcutaneously with HepG2 CTRL $^{\text {cas } 9}$ and SCARB2 ${ }^{\text {cas } 9}$ cells. (j) Representative histological (H\&E staining) images of lung metastatic tumors by in an in vivo metastasis assay. The number of metastatic lung foci detected in each group and histological staining were analyzed. (k) Numbers of metastatic lung foci in the indicated mice. 

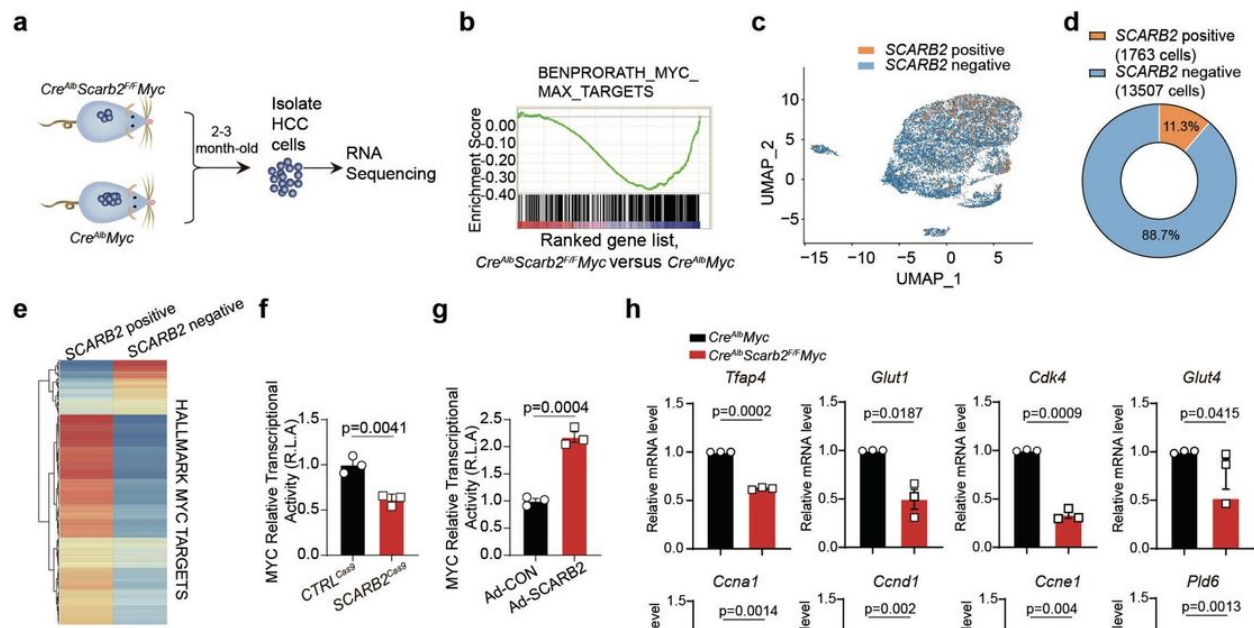

h

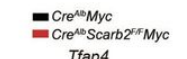

Tfap4 4 Glut1 Cak4 Glut4

i
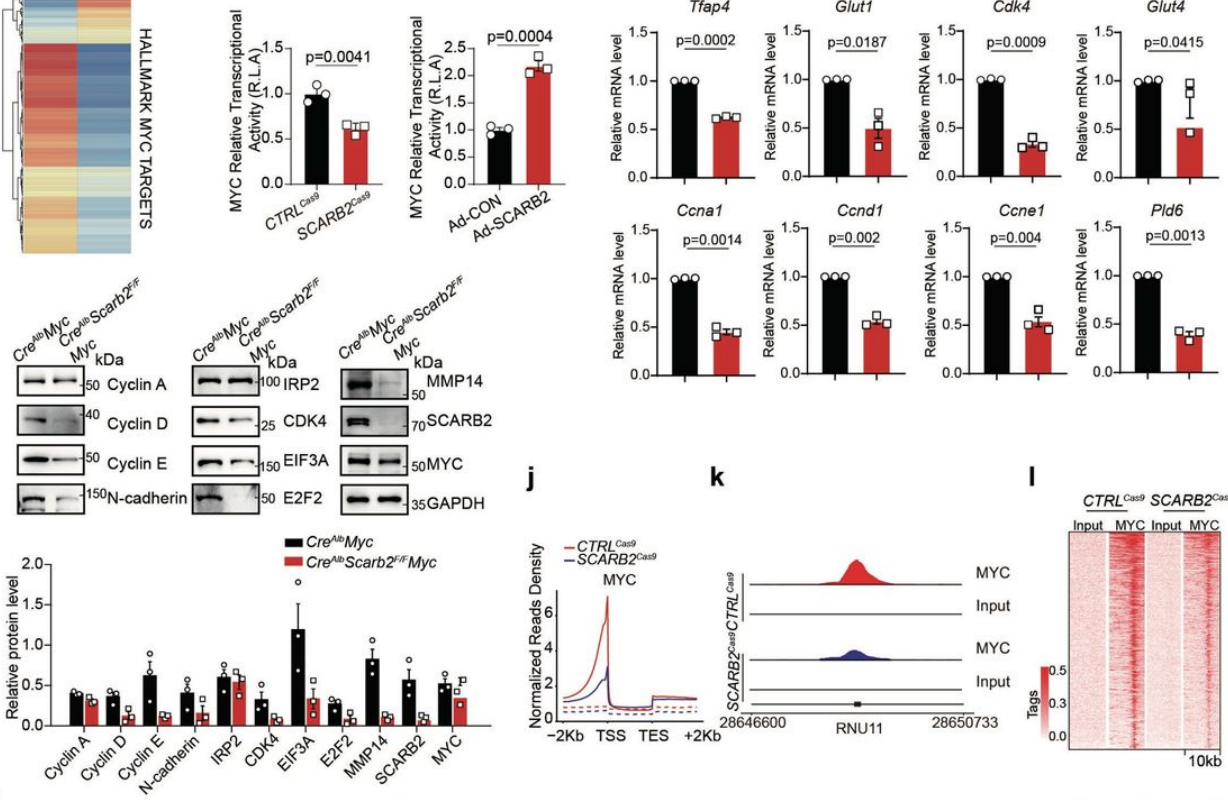

k
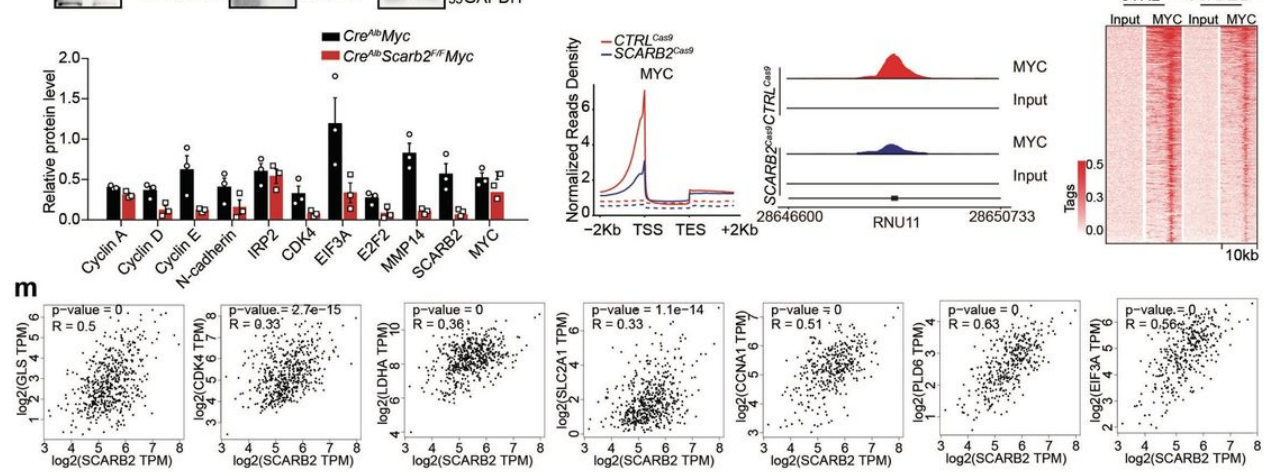

Figure 4

SCARB2 deletion inhibits MYC transcriptional activity.

(a) Approaches for RNA-seq of HCC cells isolated from $C r e^{A l b} M y c$ mice and $C r e^{A / b} S c a r b 2^{F / F} M y c$ mice. (b) GSEA results showing global downregulation of MYC target genes in the $C r e^{A / b} S c a r b 2^{F / F} M y c$ vs. Cre ${ }^{A / b} M y c$ groups. (c) Visualization of SCARB2 gene expression on different clusters of tumorspheres cultured in ultra-low attachment plates by UMAP. (d) The proportion of SCARB2 positive cells in tumorspheres nanalysed by scRNAseq. (e) Clustering and heatmap of MYC target genes in the indicated cells. ( $f$ and $g$ ) Effects of SCARB2 deletion or overexpression on the transcriptional activity of MYC. (h) The mRNA levels of MYC target genes in HCC cells from indicated mice. (i) Protein levels of MYC target genes in HCC cells from indicated mice. (j) Metagene plots of global MYC occupancy in gene bodies in CTRL ${ }^{\text {Cas9 }}$ and SCARB2 ${ }^{\text {Cas9 }}$ HCCLM3 cells. (k) ChIP-seq tracks for RNU11 in CTRL ${ }^{\text {Cas9 }}$ and SCARB2 ${ }^{\text {Cas9 }}$ HCCLM3 cells, normalized to spike-in controls. (I) Heatmap showing occupancy of genome-wide MYC peaks in $C T R L^{\text {Cas9 }}$ and $S C A R B 2^{\text {Cas9 }} \mathrm{HCCLM} 3$ cells in a $\pm 10 \mathrm{~kb}$ window surrounding the TSS. (m) Pearson's correlation between SCARB2 with MYC target genes (GLS, CDK4, LDHA, SLC2A, CCNA1, PLD6, CD133, EPCAM, or EIF3A using samples from GEPIA database. 


\section{a}

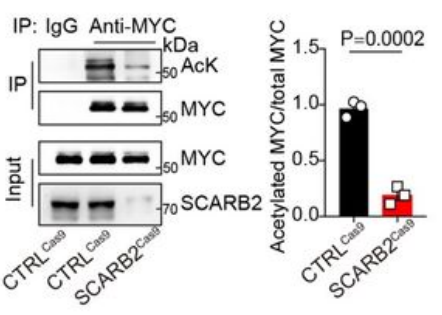

b

f

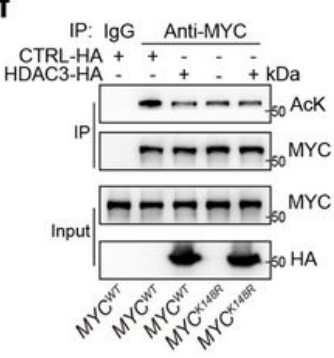

g

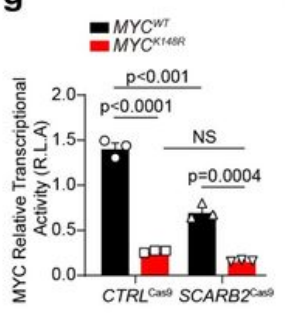

$\mathbf{k}$
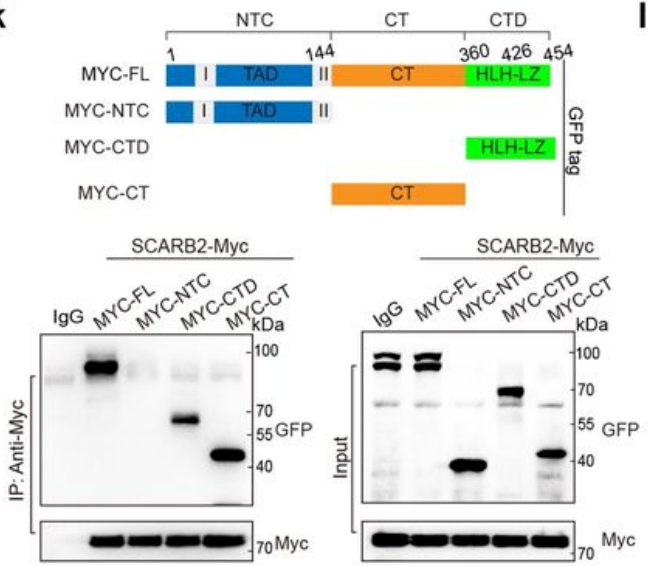

h

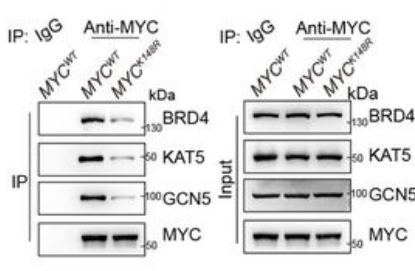

\section{I}
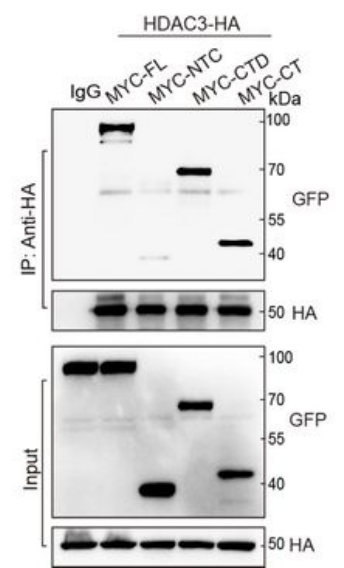

d

IgG Anti-MYC

HDAC3-HA $++;+$

CTRL-FLAG + + + + kDa

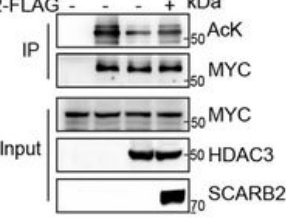

e K148R

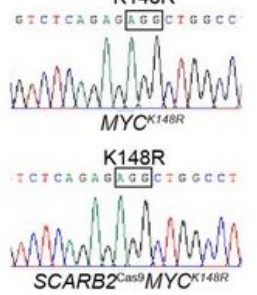

i j
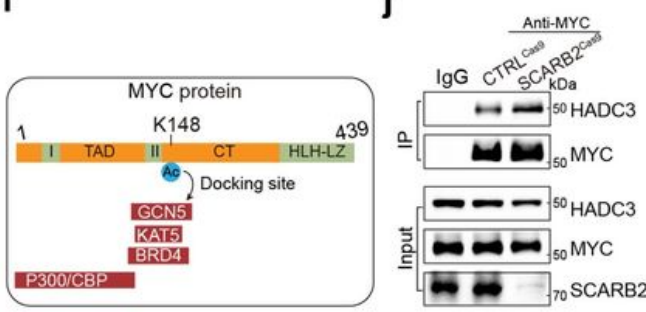

m
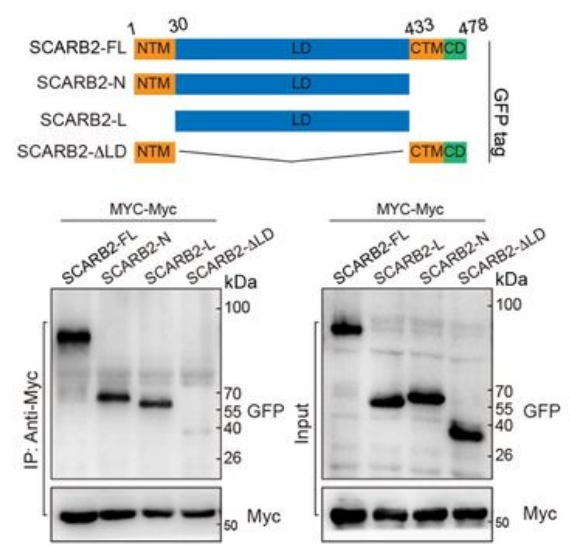

Figure 5

\section{SCARB2 interacts with MYC to disrupt HDAC3-mediated MYC deacetylation.}

(a) The effects of SCARB2 deletion on the acetylation of MYC were assessed by co-IP. (b) The effects of SCARB2 overexpression on the acetylation of MYC were assessed by co-IP. (c) Effects of SCARB2 on the acetylation of MYC K148R mutants. (d) Effect of SCARB2 overexpression on HDAC3-mediated deacetylation in vivo. (e) Sequencing verification of the codon replacement by CRISPR-Cas9 resulting in MYC (K148R). (f) Effect of HDAC3 overexpression on acetylation of MYC in $M Y C^{W T}$ and $M Y C^{K 148 R}$ HepG2 cells. (g) Effects of the MYC K148R mutation on MYC transcriptional activity in cells with or without SCARB2 depletion. (h) Effects of the K148R mutation on the interaction of MYC with BRD4, KAT5, and GCN5. (i) Schematic showing the role of MYC K148 acetylation in providing a potential docking site for binding with GCN5, KAT5, and BRD4. (j) SCARB2 deletion increased the interaction of HDAC3 and MYC. (k) Mapping of MYC regions binding to SCARB2. (I) Mapping of MYC regions binding to HDAC3. HEK 293T cells were cotransfected with the indicated constructs of MYC (GFP-tagged) and HDAC3 (HA-tagged). (m) Mapping of SCARB2 regions binding to MYC. HEK 293T cells were cotransfected with the indicated constructs of SCARB2 (Myc-tagged) and MYC (Flag-tagged). 


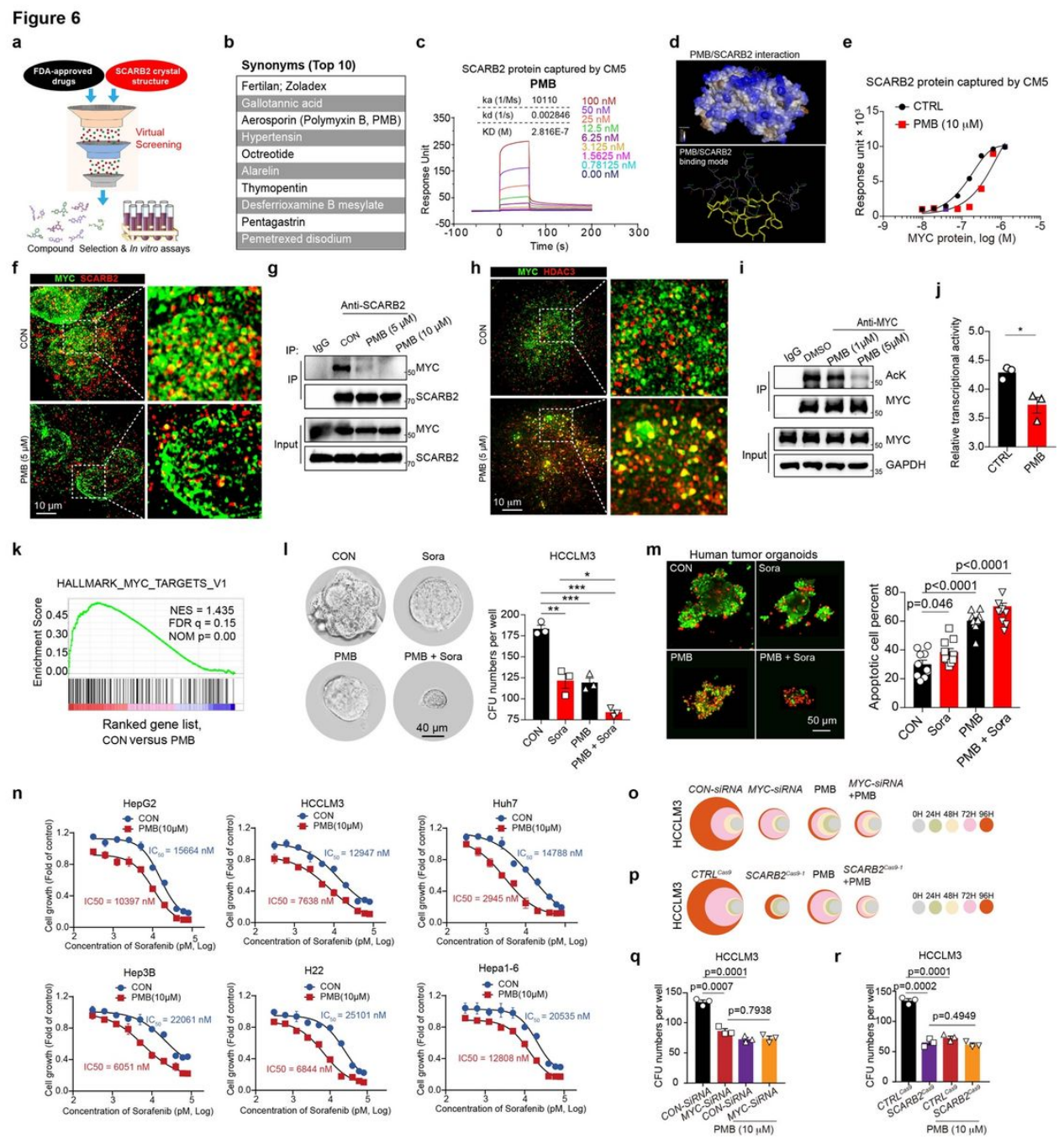

Figure 6

PMB binds with SCARB2 to suppress HCC by decreasing HDAC3-mediated MYC acetylation and MYC transcriptional activity. (a,b) Virtual screening of US FDA-approved drugs to identify small molecules binding with SCARB2 (a). The top 10 hits are listed on the right (b). (c) The kinetics of the SCARB2-PMB interaction were determined by surface plasmon resonance analysis. (d) The highest scoring docking model of the SCARB2 and PMB complex is shown. Top: surface of the PMB-SCARB2 complex. Bottom: 3D structure of the PMB (yellow)-SCARB2 complex. (e) The kinetics of the SCARB2-MYC protein interaction with or without PMB were determined by surface plasmon resonance analysis. (f) SIM images of vehicle- or PMB-treated HepG2 cells (1 hr) stained for MYC and SCARB2. (g) In vitro interaction assays showed that PMB decreased the binding of MYC and SCARB2. (h) Representative images of MYC/HDAC3 colocalization foci in HepG2 cells before and after PMB treatment. (i) Effect of PMB on MYC acetylation. (j) Transcriptional activity of MYC in HepG2 cells treated with PMB. (k) GSEA showed global downregulation of MYC target genes in PMB-treated vs. vehicle-treated HepG2 cells. (I) Quantification of tumorspheres formed by HCCLM3 cells with the indicated treatment. $(\mathrm{m})$ Analysis of the viability of human-derived HCC organoids in 3D culture following the indicated treatment. (n) Effects of PMB on sorafenib sensitivity in the indicated HCC cells. The data shown are a summary of the $\mathrm{IC}_{50}$ values for sorafenib in the indicated cell lines. (o,p) Relative viability of MYC-or SCARB2-depleted HCCLM3 cells with or without $P M B$ treatment at the indicated times. Viability was measured by a CCK-8 assay. $(\mathbf{q}, \mathbf{r})$ Quantification of tumorspheres formed by MYC-or SCARB2-depleted HCCLM3 cells with or without PMB treatment. 
Figure 7
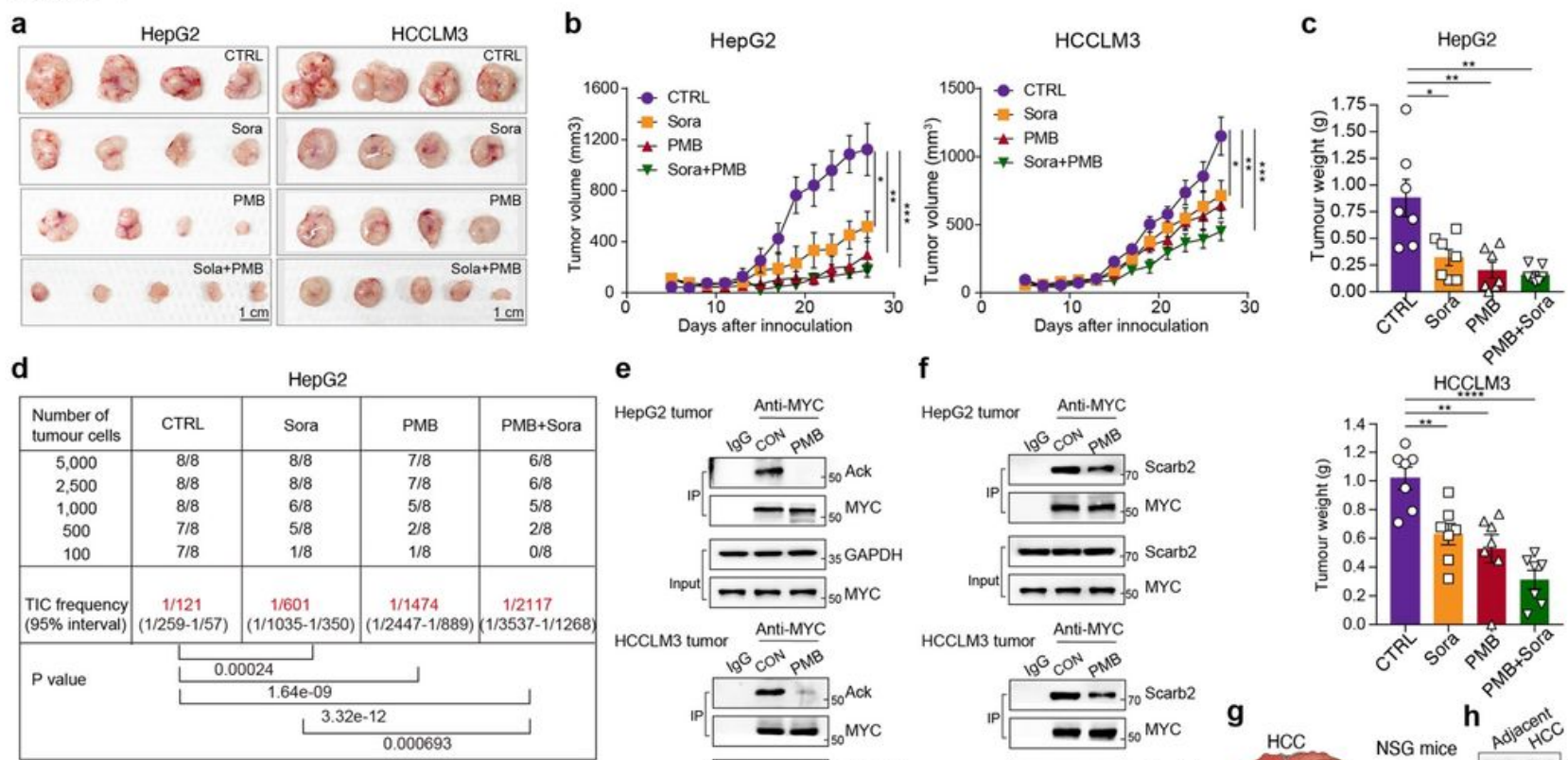

e

f
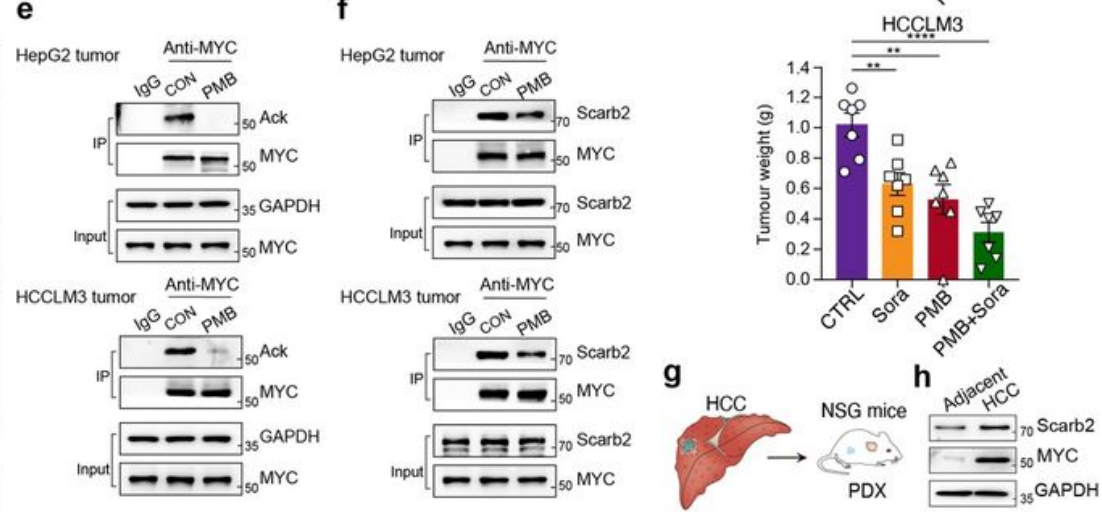

\begin{tabular}{|c|c|c|c|c|}
\hline \\
\hline $\begin{array}{l}\text { Number of } \\
\text { tumour cells }\end{array}$ & CTRL & Sora & PMB & PMB+Sora \\
\hline 5,000 & $8 / 8$ & $7 / 8$ & $7 / 8$ & $5 / 8$ \\
\hline 2,500 & $8 / 8$ & $7 / 8$ & $6 / 8$ & $4 / 8$ \\
\hline 1,000 & $8 / 8$ & $8 / 8$ & $3 / 8$ & $1 / 8$ \\
\hline 500 & $7 / 8$ & $4 / 8$ & $0 / 8$ & $0 / 8$ \\
\hline 100 & $5 / 8$ & $1 / 8$ & 0/8 & $0 / 8$ \\
\hline $\begin{array}{l}\text { TIC frequency } \\
\text { (95\% interval) }\end{array}$ & $\begin{array}{c}1 / 166 \\
(1 / 332-1 / 83)\end{array}$ & $\begin{array}{c}1 / 984 \\
(1 / 1652-1 / 586)\end{array}$ & $\begin{array}{c}1 / 2463 \\
(1 / 4152-1 / 1461\end{array}$ & $\begin{array}{c}1 / 5244 \\
1 / 9806-1 / 2804\end{array}$ \\
\hline \multirow{4}{*}{$P$ value } & \multirow{2}{*}{\multicolumn{3}{|c|}{$\begin{array}{l}1.12 \mathrm{e}-05 \\
L^{2.59 \mathrm{e}-11}\end{array}$}} & \\
\hline & & & & t \\
\hline & \multicolumn{3}{|c|}{$5.6 \mathrm{e}-16$} & \\
\hline & & & $9.63 \mathrm{e}-06$ & \\
\hline
\end{tabular}
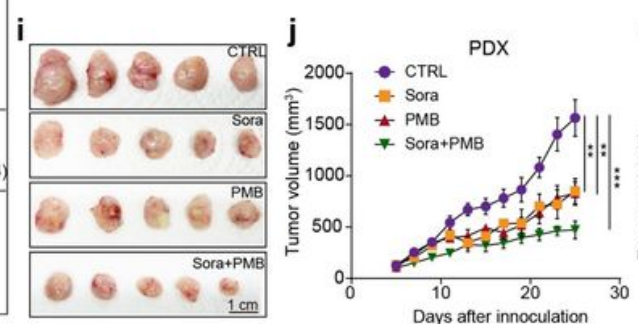

k

I
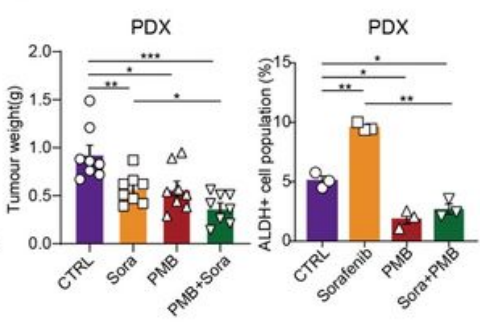

Figure 7

The combination of PMB with sorafenib synergistically suppresses HCC in CDX and PDX models.

(a) Representative images of tumors in the indicated CDX models. (b) Effects of the indicated treatments on tumor growth in the HepG2 and HCCLM3 CDX mouse models. (c) Effects of the indicated treatments on tumor weight in the indicated CDX models. (d) Effects of PMB treatment on the incidence of tumor formation from secondary transplanted HepG2 and HCCLM3 CDX-derived HCC tumors in nude mice. (e) Effects of PMB on MYC acetylation in vivo. Tumor extracts from PMB-treated mice were immunoprecipitated with an anti-MYC Ab and immunoblotted with an anti-Ack ab. (f) PMB disrupted the SCARB2-MYC interaction in vivo. Tumor lysates from mice treated as indicated were subjected to IP with an anti-MYC Ab and immunoblotted with an anti-SCARB2 Ab. (g) Strategy for establishing PDX models from HCC patients. (h) The expression of MYC and SCARB2 were examined by WB in adjacent and HCC tissues from HCC patient of PDX model. (i) Representative images of tumors in the indicated PDX models $(n=8)$. (j) Effects of the indicated treatments on tumor growth in the indicated PDX models. (k) Effects of the indicated treatments on the tumor weights in the indicated PDX models ( $\mathrm{n}=8$ per group). (I) Flow cytometry analysis for ALDH activity using the ALDEFLUOR kit in PDX model with indicated treatment.

\section{Supplementary Files}

This is a list of supplementary files associated with this preprint. Click to download.

- floatimage8.jpeg

- floatimage9.jpeg

- Onlinefloatimage10.png

- floatimage11.jpeg

- floatimage12.jpeg

- floatimage13.jpeg

- floatimage14.jpeg 
- SupplementaryTable1.docx

Page 21/21 